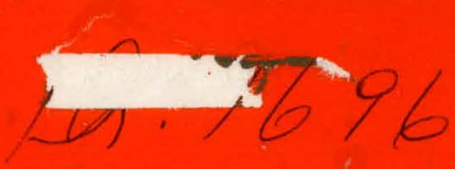

\title{
SERAPH Facility Capabilities
}

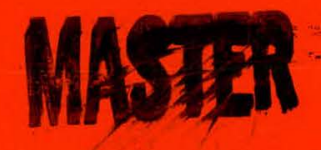

James Castle

Wen Su
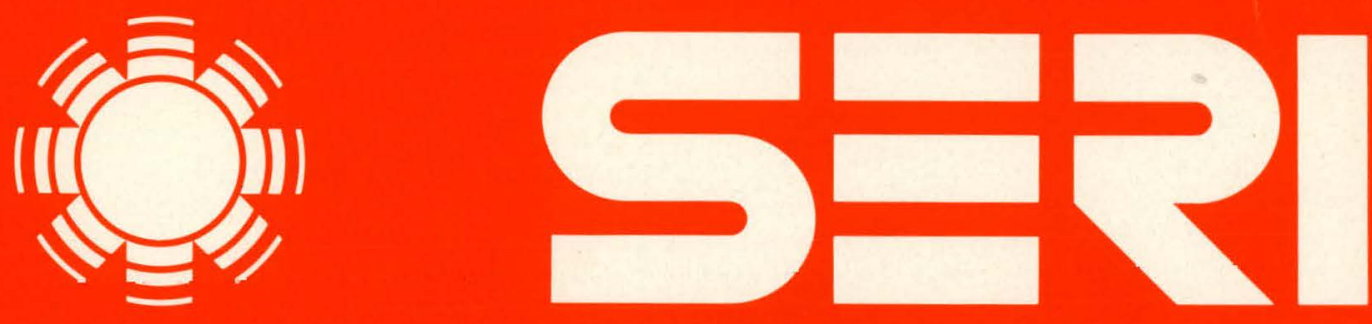

Solar Energy Research Institute

A Division of Midwest Research Institute

1617 Cole Boulevard

Golden, Colorado 80401

Operated for the

U.S. Department of Energy

under Contract No. EG-77-C-01-4042 


\section{DISCLAIMER}

This report was prepared as an account of work sponsored by an agency of the United States Government. Neither the United States Government nor any agency Thereof, nor any of their employees, makes any warranty, express or implied, or assumes any legal liability or responsibility for the accuracy, completeness, or usefulness of any information, apparatus, product, or process disclosed, or represents that its use would not infringe privately owned rights. Reference herein to any specific commercial product, process, or service by trade name, trademark, manufacturer, or otherwise does not necessarily constitute or imply its endorsement, recommendation, or favoring by the United States Government or any agency thereof. The views and opinions of authors expressed herein do not necessarily state or reflect those of the United States Government or any agency thereof. 


\section{DISCLAIMER}

Portions of this document may be illegible in electronic image products. Images are produced from the best available original document. 
Printed in the United States of America Available from:

National Technical Information Service

U.S. Department of Commerce

5285 Port Royal Road

Springfield, VA 22161

Price:

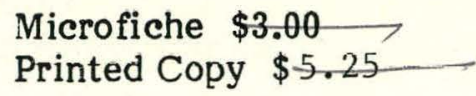

\begin{abstract}
NOTICE
This report was prepared as an account of work sponsored by the United States Government. Neither the United States nor the United States Department of Energy, nor any of their employees, nor any of their contractors, subcontractors, or their employees, makes any warranty, express or implied, or assumes any legal liability or responsibility for the accuracy, completeness or usefulness of any information, apparatus, product or process disclosed, or represents that its use would not infringe privately owned rights.
\end{abstract}


SERI /RR $-632-702$

UC CATEGORY: UC-59b

SERAPH FACILITY CAPABILITIES

JAMES CASTLE

WEN SU

JUNE 1980

PRePARED UNder TAGK NO. 3471.10

\section{Solar Energy Research Institute}

A Division of Midwest Research Institute

1617 Cole Boulevard

Golden, Colorado 80401

Prepared for the

U.S. Department of Energy

Contract No. EG-77-C-01-4042

DISCLAIMER

This book was prepared as on account of work sponsored by an agency of tha United States Government, Neither the United Staies Governiment nor any agency thereot, nor any of their employees, makes any

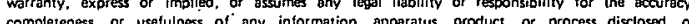

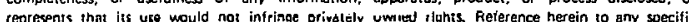
commercial product, process, or service by trade name, irademark, manutactures, or olherwise. does not necessarily constitute or imply its endorsement, recommendation, or favoring by the Unites States Government or bny agancy thereef. The viaws and opinions of authors expressed herein do nol 


\section{THIS PAGE \\ WAS INTENTIONALLY \\ LEFT BLANK}


: SEP 1

PREFACE

This document describes work done on Subtask 3471.10 within the Industrial Process Heat Program at SERI. This subtask is concerned with the design, construction, and documentation pertaining to SERAPH (Solar Energy Research and Applications in Process Heat), a system test and evaluation facility to be erected near Golden, Colo. The capabilities of this facility are described herein.
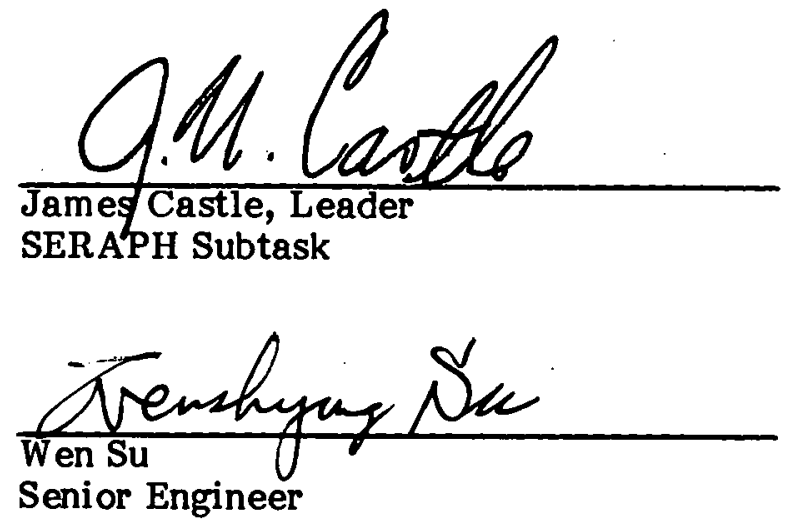

Approved for:

SOLAR ENERGY RESEARCH INSTITUTE

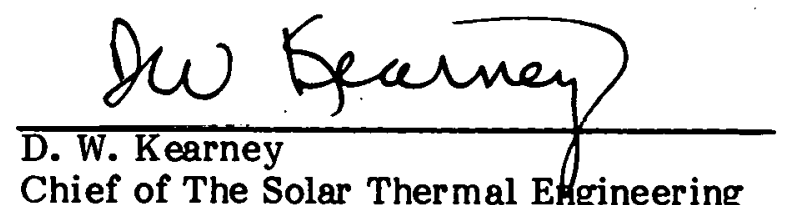

Chief of The Solar Thermal Engineering

Development Branch

Manager of The Solar Thermal Ocean and Wind Division

iii 


\section{THIS PAGE}

\section{WAS INTENTIONALLY \\ LEFT BLANK}




\section{SUMMARY}

\section{OBJECTIVE}

The objective of this report is to provide a detailed description of the SERAPH (Solar Energy Research and Applications in Process Heat) facility. This facility will be used to investigate technical issues pertinent to the application of solar energy within industry for the purpose of satisfying midtemperature (less than $300^{\circ} \mathrm{C}$ ) process heat requirements. SERAPH will be erected at the Solar Energy Research Institute's Field Test Site near Golden, Colo.

\section{DISCUSSION}

Industries within the United States consume a large portion of the nation's yearly energy budget, and a significant fraction of this consumption takes place at low and intermediate temperatures. Several existing solar technologies may be readily adaptable to energy delivery at these temperatures. Specifically, line-focus concentrators, seasonally adjusted concentrators, and flat-plate and tubular collectors may find application along with thermal storage devices in some instances. However, a number of engineering questions exist regarding the extent to which solar systems can operate reliably and in a controlled fashion over long periods within industry when subjected to specific load demands. Such questions apply most of ten to the application of concentrating collectors.

The SERAPH facility is designed so that a series of solar systems can be deployed on a small scale and have their operating characteristics accurately monitored in a closely controlled environment. Emphasis will be on evaluating complete systems as they respond to artificial loads programmed by the facility operators. It will be possible to reconfigure the facility quickly so that a series of technical concerns can be addressed while employing a number of solar-related components. Provision will exist for providing supplem ental, fossil fuel-derived energy to the solar systems when necessary, as would be the case within industry.

Design assembly, procurement, and operational procedures at SERAPH will mimic to the maximum extent possible those routinely used by industrialists. Industry visitors with an interest in solar technologies and their application will be invited to observe and review facility operations. Technical results will be widely disseminated via SERI reports and data base entries.

\section{CONCLUSIONS}

The SERAPH facility at SERI will provide a comprehensive investigative tool applicable to solar system technologies in a form useful for industry. It will serve to expand the engineers' and designers' knowledge of system behavior and thereby enable this technology to find acceptance within the industry. The facility will be flexible in its layout so that a variety of system configurations can be readily introduced for investigation and evaluation. Results will be used to verify computer analysis predictions and will be distributed to interested parties through regular reports. 
THIS PAGE

\section{WAS INTENTIONALLY \\ LEFT BLANK}




\section{TABLE OF CONTENTS}

Page

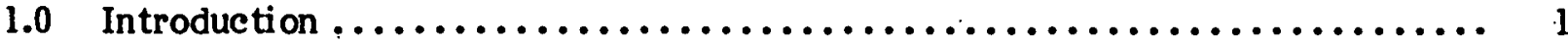

2.0 Facility Objectives $\ldots \ldots \ldots \ldots \ldots \ldots \ldots \ldots \ldots \ldots \ldots \ldots \ldots \ldots \ldots \ldots \ldots \ldots \ldots$

$3.0 \quad$ Field Test Site $\ldots \ldots \ldots \ldots \ldots \ldots \ldots \ldots \ldots \ldots \ldots \ldots \ldots \ldots \ldots \ldots \ldots \ldots \ldots \ldots \ldots$

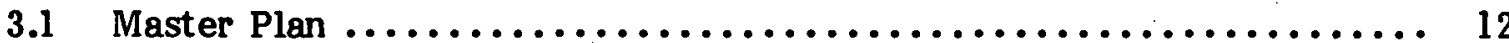

3.2 SERAPH Site $\ldots \ldots \ldots \ldots \ldots \ldots \ldots \ldots \ldots \ldots \ldots \ldots \ldots \ldots \ldots \ldots \ldots \ldots \ldots$

$4.0 \quad$ EDS-Main Features $\ldots \ldots \ldots \ldots \ldots \ldots \ldots \ldots \ldots \ldots \ldots \ldots \ldots \ldots \ldots \ldots \ldots$

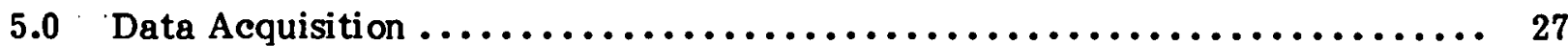

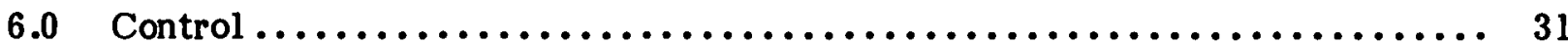

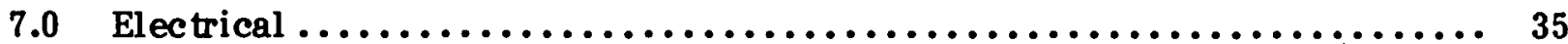

8.0 Hazards/Safety $\ldots \ldots \ldots \ldots \ldots \ldots \ldots \ldots \ldots \ldots \ldots \ldots \ldots \ldots \ldots \ldots \ldots \ldots \ldots \ldots . \ldots \ldots$

$9.0 \quad$ Facility Uses $\ldots \ldots \ldots \ldots \ldots \ldots \ldots \ldots \ldots \ldots \ldots \ldots \ldots \ldots \ldots \ldots \ldots \ldots \ldots \ldots \ldots$

9.1 Solar Energy Delivery System ........................... 41

9.2 Commercial Chiller Evaluation........................ 42

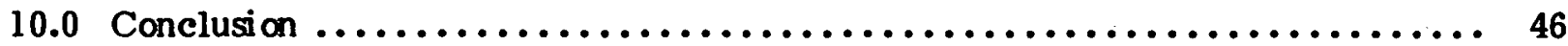




\section{LIST OF FIGURES}

Page

1-1 Proposed SERAPH F acility $\ldots \ldots \ldots \ldots \ldots \ldots \ldots \ldots \ldots \ldots \ldots \ldots \ldots \ldots \ldots \ldots \ldots \ldots$

2-1 IPH System Elements $\ldots \ldots \ldots \ldots \ldots \ldots \ldots \ldots \ldots \ldots \ldots \ldots \ldots \ldots \ldots \ldots \ldots \ldots$

3-1 SERI Field Test Site Plan $\ldots \ldots \ldots \ldots \ldots \ldots \ldots \ldots \ldots \ldots \ldots \ldots \ldots \ldots \ldots \ldots \ldots$

3-2 Field Test Site Buff $e \ldots \ldots \ldots \ldots \ldots \ldots \ldots \ldots \ldots \ldots \ldots \ldots \ldots \ldots \ldots \ldots \ldots \ldots \ldots \ldots$

3-3 The Organizational Grid................................ 16

3-4 SERAPH Facility Support Elements-Plan View................. 17

4-1 EDS Elements $\ldots \ldots \ldots \ldots \ldots \ldots \ldots \ldots \ldots \ldots \ldots \ldots \ldots \ldots \ldots \ldots \ldots \ldots \ldots \ldots \ldots$

$4-2$ Area 20 P\&ID $\ldots \ldots \ldots \ldots \ldots \ldots \ldots \ldots \ldots \ldots \ldots \ldots \ldots \ldots \ldots \ldots \ldots \ldots \ldots \ldots \ldots$

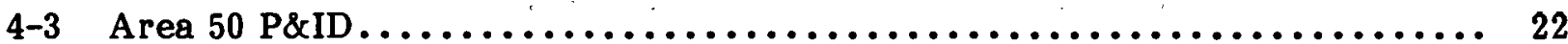

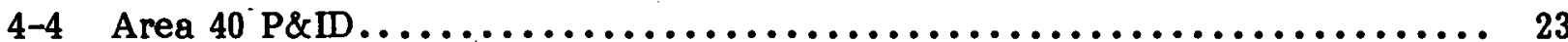

4-5 Interconnections Between Areas 20,40 , and $50 \ldots \ldots \ldots \ldots \ldots \ldots \ldots \ldots \ldots$

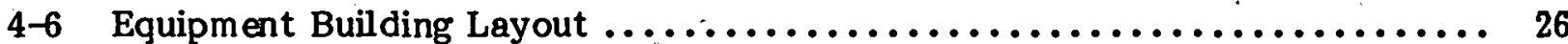

5-1 Data Acquisition System Block Diagram ..................... 28

$6-1$ Control House Layout................................ 31

6-2 Control System Block Diagram $\ldots \ldots \ldots \ldots \ldots \ldots \ldots \ldots \ldots \ldots \ldots \ldots \ldots \ldots \ldots$

6-3 Typical Control Loop $\ldots \ldots \ldots \ldots \ldots \ldots \ldots \ldots \ldots \ldots \ldots \ldots \ldots \ldots \ldots \ldots \ldots \ldots \ldots \ldots$

7-1 Electrical One-Line Program $\ldots \ldots \ldots \ldots \ldots \ldots \ldots \ldots \ldots \ldots \ldots \ldots \ldots \ldots \ldots$

9-1 SEDS Piping \& Instrumentation Diagram..................... 43

9-2 Collector Field Layout - Plan View ......................... 44

9-3 Chiller Test Configuration............................. 45 
$n^{*}$

GED in

RR-702

LIST OP TABLES

Page

3-1 Summary of Monthly Precipitation Data for Lakewood, Colo............. 9 9

3-2 Summary of Monthly Temperature for Lakewood, Colo................ 10

3-3 Summary of Monthly Average Humidity for Rocky Flats, Colo.

11

3-4 Sunshine and Cloudiness at Denver, Colo..................... 12

3-5 Field Test Site Master Plan Goals $\ldots \ldots \ldots \ldots \ldots \ldots \ldots \ldots \ldots \ldots \ldots \ldots \ldots$

3-6 Master Plan Concepts................................ 14

3-7 Utilities Available at the SERAPH Site..................... 18

4-1 Energy Distribution System (EDS) Capabilities $\ldots \ldots \ldots \ldots \ldots \ldots \ldots \ldots \ldots$

ix 


\section{SECTION 1.0}

\section{INTRODUCTION}

The Solar Energy Research Institute (SERI) is developing the SERAPH (Solar Energy Research and Applications in Process Heat) facility at its Field Test Site near Golden, Colo. SERA $\overline{P H}$ is to be a versatile outdoor testing and evaluation installation devoted to the application of solar thermal energy to industrial process heat (IPH) requirements (Fig. 1-1). Design, development, and operation of the facility is funded by the U.S. Department of Energy (DOE) through the SERI Solar Thermal Program. SERI is responsible for experimental design, operation, and reporting at SERAPH. Unique operational and testing features (e.g., modular, flexible, and programmable thermal loads) exist within SERAPH with respect to other solar installations.

It is SERI's intention to assemble the major elements of various IPH solar systems at SERAPH for evaluation. Technical issues will be addressed that currently restrict the widespread use of solar energy in industry. As the technology matures, major equipment changes will take place and new technical topies will be addressed. Entire collector fi elds (100-300 $\mathrm{m}^{2}$ in aperture), storage devices, and individual components will be moved in and out of the facility. Theref ore a key aspect of SERAPH will be its flexibility. The supporting capabilities that will be unchanged at SERAPH will include data acquisition and process control electronics, power distribution gear, the auxiliary energy source, fluid transfer pumps, and control valves. A process load simulation package will be available to impose representative thermal loads on the system being evaluated. No fixed energy requirements will exist that are to be satisfied via solar collection unless convenient to do so. Collected energy can ultimately be discharged to the atmosphere via a wet cooling tower.

SERAPH will be accessible to the public. Potential IPH solar system users will be able to observe firsthand the equipment and controls that they might be considering. The results of studies performed at the facility will be widely available through the nationwide Solar Energy Inf ormation Data Base.

The sections of this report describe SERAPH in detail. Items that are covered include site description, data acquisition and control, experimental operations, equipment description, safety and hazards, and planned uses. The objective of this report is to provide a sufficiently thorough facility description so that interested parties can be aware of the type of results to be expected from facility operations, and so that those proposing activities are aware of the facility capabilities and constraints. Communication regarding SERAPH should be through the SERI Solar Thermal Program Manager. 


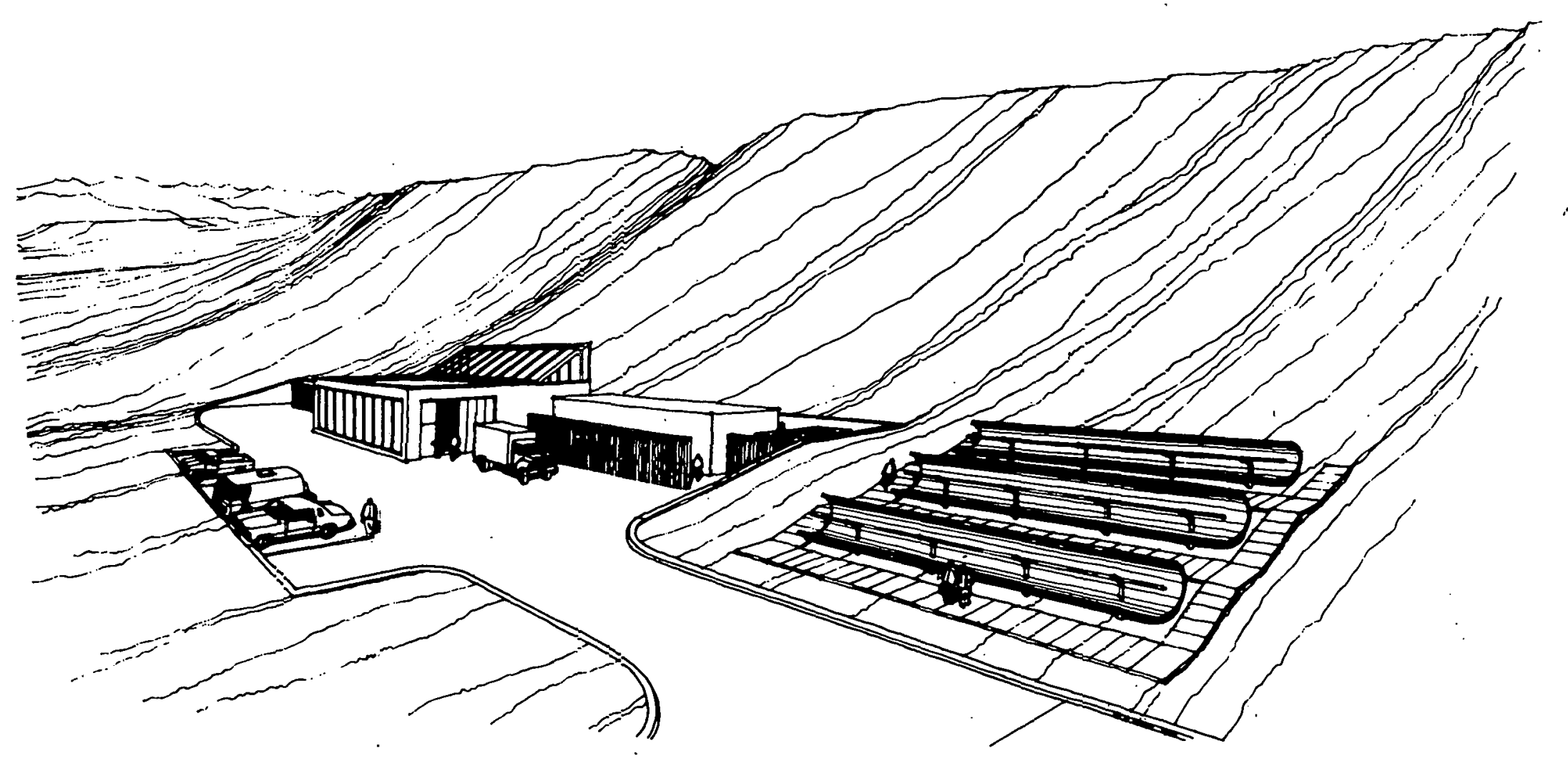

Figure 1-1. Proposed SERAPH Facilily 


\section{SECTION 2.0}

\section{PACIITYY OBJECTIVES}

SERAPH is a major element of the SERI Solar Thermal Program. Its purpose is to further the acceptance and application of solar thermal energy systems in meeting the process heat requirements of industry. This is important because the U.S. industrial market is the nation's largest consumer of nonrenewable fossil fuels. The process heat used by industry is about $25 \%$ of national energy consumption. The Solar Thermal Program at SERI addresses those technical, institutional, market, and economic issues that currently inhibit the widespread use of solar energy by industry. The use of solar systems by industry appears attractive because of year-round usage, potential economics of scale, the presence of trained technical personnel, and the fact that a large energy demand exists in the mid- and low-temperature $\left(\leqslant 300^{\circ} \mathrm{C}\right)$ range. Consequently, SERI is responsible for a significant eff ort to displace conventional industrial fuels with solar energy. The SERI program operates in conformance with fossil fuel energy displacement goals established by the Department of Energy, and program tasks are coordinated with related work at other national and private laboratories.

SERAPH will assist in achieving the Solar Thermal Program by addressing technical issues pertinent to the analysis, design, operation, and maintenance of solar systems as they would be configured within industry. Emphasis will be placed on the system-evaluative nature of the facility in contrast to single-component evaluation (individual components will normally be well characterized before being introduced into the facility). The major elements of a typical IPH system are shown in Fig. 2-1. SERAPH will be concerned with maximizing the efficiency and dependability of the interaction of these elements. The facility layout is modular in nature, and it will be possible to modify or replace the major elements.

Investigations at SERAPH will mimic industrial contexts extensively. Trained O\&M personnel will be on hand, and system use will be repetitive and intensive. Established industrial practices with respect to equipment quality, assembly techniques, codes, and standards (e.g., National Electrical Code, ANSI Pressure Piping Standard, FM approval, ISA standards) will be adhered to where applicable. Industrial personnel will be brought to the facility periodically to verify the appropriateness of this guiding philosophy.

Information that will be generated at the SERAPH facility-test summaries, design tools, operational procedures, maintenance requirements-will only be useful if conveyed to potential users. Several mechanisms will be employed to assure timely information dissemination. The SERI Information Systems Division carries major responsibility for this critical educational function. Regular technical reports, subject bibliographies, industry and trade workshops, facility visits, and media presentations are the major avenues for announcing Institute accomplishments. Numerical and bibliographic materials are available nationwide through the Solar Energy Information Data Base (SEIDB), which is built and maintained by the SERI Information Systems Division: Eventually, analysis models will be available through SEIDB also. 


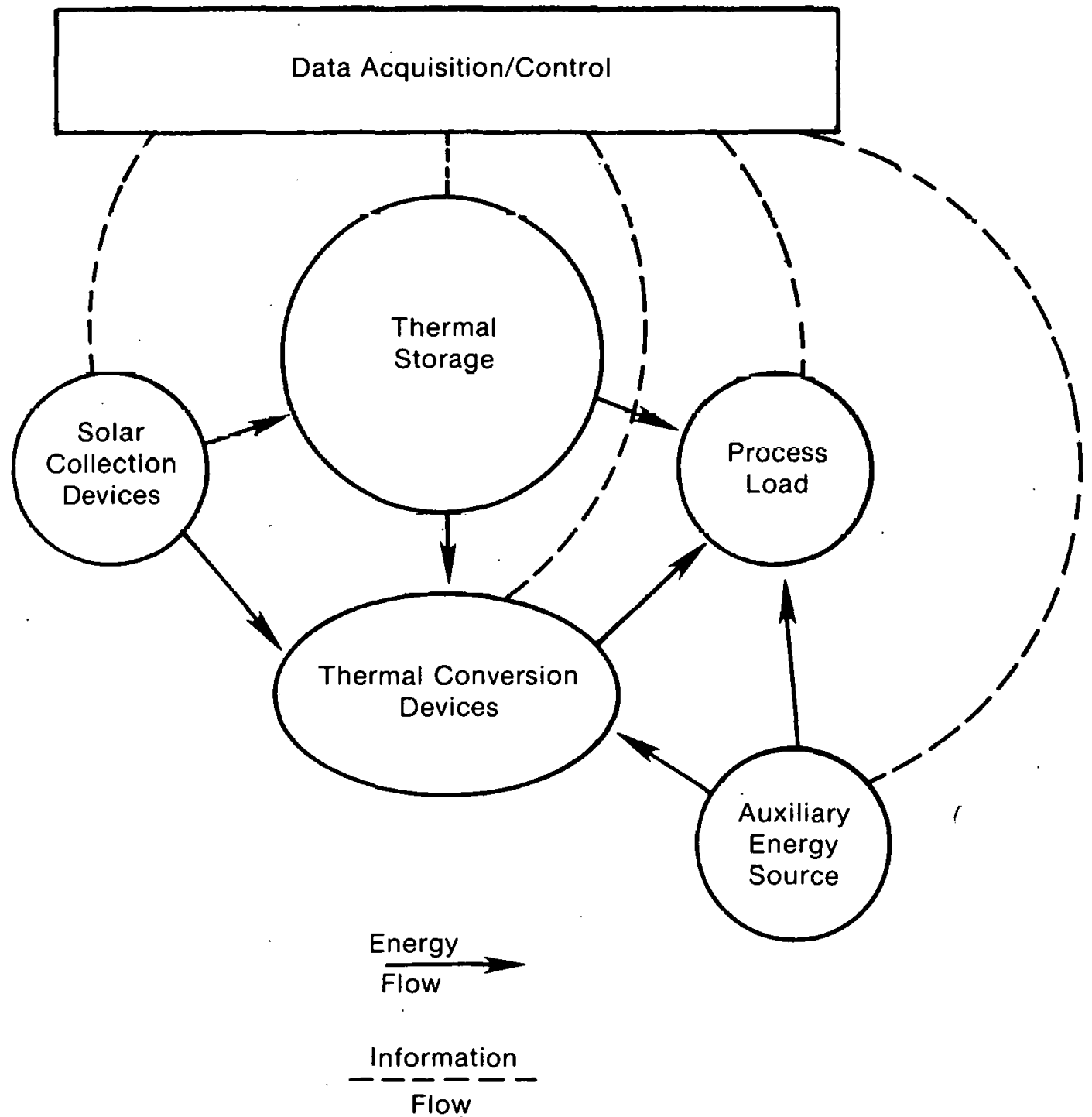

Figure 2-1. I PH Systom Elements

A series of specific SERAPH objectives exists within the framework of the overall program objectives. The primary objectives are:

- to create, on a small scale, industrial process heat systems;

- to validate analytical models that compute system performance characteristics;

- to develop controls to satisfactorily manipulate the collector field, auxiliary energy addition, thermal storage, and load; 
든

- to verify the performance of specific components and materials in a system environment;

- to provide a major experimental capability and experience at SERI;

- to establish the reliability of critical elements;

- to exercise failure modes;

- to establish O\&M needs; and

- to investigate thermal conversion technology.

SERAPH's secondary objectives include:

- training industrial personnel;

- stimulating public/legislative interest in solar IPH; and

- investigating speculative technical issues pertaining to IPH solar systems.

The SERAPH facility will provide an environment superior to that of the industrial field experiments being funded by DOE for accomplishing these primary and secondary goals. The unique advantages of SERAPH vis-à-vis the field experiments will be:

- trained personnel on continuous duty;

- flexibility due to modular construction;

- more sophisticated data acquisition and control features;

- no dependence on process load;

- less public visibility when needed for exploratory investigations of unconfirmed technology; and

- superior documentation and information dissemination.

Realization of SERAPH objectives will help resolve technical uncertainties that are presently a problem with decision makers in industry. Results and conclusions can then receive large-scale verification in the DOE field experiments, in which SERI participates and exercises a portion of the technical management role. 


\section{Sミㅋ*}




\section{SECTION 3.0}

\section{FIELD TEST SITE}

The SERAPH facility will be located on a 2-1/2 acre (about 10,000- $\mathrm{m}^{2}$ ) parcel at the SERI Field Test Site near Golden, Colo. The Field Test Site is adjacent to the land on which the SERI Permanent Offices will be erected and is within two miles of the present office complex. Figure 3-1 describes the entire Field Test Site and indicates the geographical relationship of SERAPH to the other site activities. The site is on the lower south-facing slope of South Table Mountain at an elevation of 5900 feet $(1800 \mathrm{~m})$. Latitude is $39^{\circ} 50^{\prime} \mathrm{N}$, and longitude is $105^{\circ} 15^{\prime} \mathrm{W}$.

Several sources exist for meteorological records to indicate the weather to be expected at the Field Test Site. These records have been generated at Denver, Lakewood, Rocky Flats, and Boulder, Colo. Tables 3-1 and 3-2 detail precipitation and temperature on a monthly basis for Lakewood (three miles east of the site). Humidity records in Table 3-3 are from Rocky Flats (approximately 8 miles north of the site). The sunshine and cloudiness records in Table 3-4 were taken at Stapleton International Airport in Denver. Additional solar radiation data are available from the National Atmospheric and Oceanographic Administration in Boulder. Conditions in Boulder are probably more representative of Golden than are those of Denver because of Boulder's similar relationship to the Front Range of the Rocky Mountains (cloud conditions are strongly dependent on distançe from the Front Range). Average daily insolation in Boulder is 15,500 to $16,000 \mathrm{~kJ} / \mathrm{m}^{2}$. Sky conditions for the spring behave rather regularly; most days start clear, cloud over by late morning or early afternoon, and a rainshower may follow. Spring is the cloudiest season and fall is the sunniest. An insolation data base is now being generated by SERI using measurements at the Field Test Site. Weather predictions are provided to SERI directly from a local meteorological firm.

Westerly Chinook winds (which are characteristic of the Front Range) can subject the site to strongly turbulent wintertime winds in excess of $100 \mathrm{mph}(13.5 \mathrm{~m} / \mathrm{s})$. 


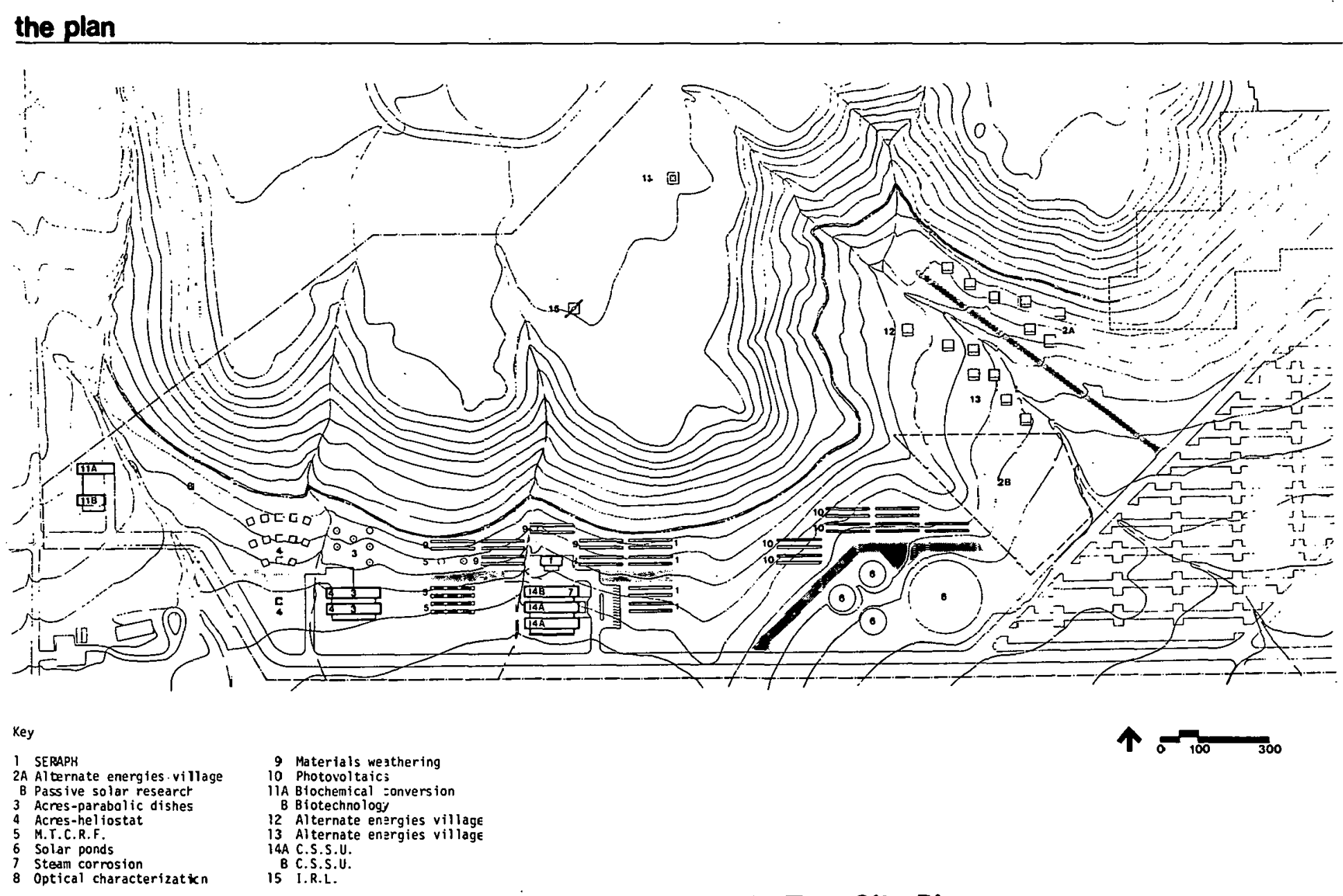

Figure 3-1. SERI Field Test Site Plan

(10-1t. contours shown) 
Table 3-1. SUMMARY OP MONTHLY PRECIPITATION DATA FOR LAKEWOOD, COLO. ${ }^{a}$

\begin{tabular}{|c|c|c|c|c|c|c|c|c|c|c|c|c|c|c|}
\hline & & Jan. & Feb. & Mar. & Apr. & May & Jun. & Jul. & Aug. & Sept. & Oct. & Nov. & Dec. & Annuel \\
\hline $\begin{array}{l}\text { Monthly Precipitation } \\
\text { (in) }\end{array}$ & $\begin{array}{r}\text { Avg. } \\
\text { Max. } \\
\text { Min. } \\
\text { Months of Recorc }\end{array}$ & $\begin{array}{l}0.51 \\
1.35 \\
0.05 \\
13\end{array}$ & $\begin{array}{l}0.53 \\
1.07 \\
0.04 \\
13\end{array}$ & $\begin{array}{l}1.16 \\
3.38 \\
0.14 \\
13\end{array}$ & $\begin{array}{l}1.67 \\
3.64 \\
0.00 \\
13\end{array}$ & $\begin{array}{l}2.21 \\
6.77 \\
0.20 \\
13\end{array}$ & $\begin{array}{l}2.15 \\
5.09 \\
0.45 \\
13\end{array}$ & $\begin{array}{l}2.04 \\
5.17 \\
0.46 \\
13\end{array}$ & $\begin{array}{l}1.16 \\
2.62 \\
0.23 \\
14\end{array}$ & $\begin{array}{l}1.42 \\
3.25 \\
0.33 \\
14\end{array}$ & $\begin{array}{l}0.95 \\
3.71 \\
0.05 \\
14\end{array}$ & $\begin{array}{l}0.87 \\
2.82 \\
0.16 \\
14\end{array}$ & $\begin{array}{l}0.48 \\
1.64 \\
0.07 \\
14\end{array}$ & 15.16 \\
\hline $\begin{array}{l}\text { Greatest Daily } \\
\text { Precipitation } \\
\text { (in) }\end{array}$ & $\begin{array}{r}\text { Amount } \\
\text { Year and Day } \\
\text { Months of Record }\end{array}$ & $\begin{array}{l}1.04 \\
197418 \\
13\end{array}$ & $\begin{array}{r}0.62 \\
197120 \\
13\end{array}$ & $\begin{array}{c}1.40 \\
196312 \\
13\end{array}$ & $\begin{array}{r}2.33 \\
196713 \\
12\end{array}$ & $\begin{array}{r}2.70 \\
196907 \\
13\end{array}$ & $\begin{aligned} 2.77 \\
196316 \\
13\end{aligned}$ & $\begin{array}{c}1.91 \\
197517 \\
13\end{array}$ & $\begin{array}{r}2.00 \\
197513 \\
14\end{array}$ & $\begin{array}{l}1.52 \\
197022 \\
14\end{array}$ & $\begin{array}{r}0.95 \\
1974 \quad 12 \\
14\end{array}$ & $\begin{array}{r}1.36 \\
197509 \\
14\end{array}$ & $\begin{array}{r}0.43 \\
197319 \\
14\end{array}$ & \\
\hline Monthly Snowfall (in) & $\begin{array}{r}\text { Avg. } \\
\text { Max. } \\
\text { Min. } \\
\text { Montins of Record }\end{array}$ & $\begin{array}{r}6.4 \\
12.0 \\
1.0 \\
11\end{array}$ & $\begin{array}{r}7.9 \\
20.6 \\
0.0 \\
11\end{array}$ & $\begin{array}{r}14.0 \\
38.7 \\
3.0 \\
11\end{array}$ & $\begin{array}{r}9.8 \\
27.7 \\
0.0 \\
11\end{array}$ & $\begin{array}{l}0.7 \\
5.0 \\
0.0 \\
13\end{array}$ & $\begin{array}{c}0.0 \\
0.0 \\
0.0 \\
13\end{array}$ & $\begin{array}{l}0.0 \\
0.0 \\
0.0 \\
14\end{array}$ & $\begin{array}{l}0.0 \\
0.0 \\
0.0 \\
14\end{array}$ & $\begin{array}{c}0.6 \\
5.5 \\
0.0 \\
13\end{array}$ & $\begin{array}{c}4.8 \\
33.0 \\
0.0 \\
14\end{array}$ & $\begin{array}{r}9.9 \\
36.0 \\
2.0 \\
13\end{array}$ & $\begin{array}{r}9.3 \\
27.0 \\
0.0 \\
12\end{array}$ & 63.3 \\
\hline $\begin{array}{l}\text { Greatest Depth of } \\
\text { Snow on } \\
\text { Ground in } \\
\text { Month }\end{array}$ & $\begin{array}{r}\text { (in) } \\
\text { Year and Day } \\
\text { Months of Record }\end{array}$ & $\begin{array}{c}9 . \\
197406+ \\
12\end{array}$ & $\begin{array}{l}14 . \\
196515 \\
11\end{array}$ & $\begin{array}{l}19 . \\
197013 \\
13\end{array}$ & $\begin{array}{l}16 . \\
197001 \\
12\end{array}$ & $\begin{array}{l}5 . \\
197301 \\
12\end{array}$ & $\frac{0}{13}$ & $\frac{0}{14}$ & $\frac{0}{14}$ & $\begin{array}{l}11 . \\
197117 \\
14\end{array}$ & $\begin{array}{l}15 . \\
196904 \\
12\end{array}$ & $\begin{array}{c}16 . \\
197202 \\
12\end{array}$ & $\begin{array}{l}9 . \\
197210\end{array}$ & \\
\hline $\begin{array}{l}\text { No. Days Precipitation } \\
\text { Greater Than } \\
\text { or Equal to } 0.1 \text { in }\end{array}$ & $\begin{array}{r}\text { Avg. } \\
\text { Max. } \\
\text { Min. } \\
\text { Months of Record }\end{array}$ & $\begin{array}{c}1.6 \\
3 \\
0 \\
-13\end{array}$ & $\begin{array}{l}1.8 \\
4 \\
0 \\
13\end{array}$ & $\begin{array}{c}3.7 \\
10 \\
1 \\
13\end{array}$ & $\begin{array}{l}3.9 \\
9 \\
0 \\
13\end{array}$ & $\begin{array}{l}4.2 \\
9 \\
1 \\
13\end{array}$ & $\begin{array}{l}3.8 \\
10 \\
1 \\
13\end{array}$ & $\begin{array}{l}4.6 \\
10 \\
1 \\
13\end{array}$ & $\begin{array}{l}3.1 \\
7 \\
1 \\
14\end{array}$ & $\begin{array}{l}3.6 \\
7 \\
1 \\
14\end{array}$ & $\begin{array}{l}2.7 \\
10 \\
0 \\
14\end{array}$ & $\begin{array}{l}2.6 \\
5 \\
1 \\
14\end{array}$ & $\begin{array}{l}1.9 \\
4 \\
0 \\
14\end{array}$ & 37.7 \\
\hline $\begin{array}{l}\text { No. Days Precipitation } \\
\text { Greater Than } \\
\text { or Equal to } 0.5 \text { in }\end{array}$ & $\begin{array}{r}\text { Avg. } \\
\text { Max. } \\
\text { Min. } \\
\text { Months of Record }\end{array}$ & $\begin{array}{l}0.2 \\
1 \\
0 \\
13\end{array}$ & $\begin{array}{c}0.1 \\
1 \\
0 \\
13\end{array}$ & $\begin{array}{l}0.5 \\
3 \\
0 \\
13\end{array}$ & $\begin{array}{c}1.0 \\
3 \\
0 \\
13\end{array}$ & $\begin{array}{l}1.5 \\
5 \\
0 \\
13\end{array}$ & $\begin{array}{l}1.2 \\
3 \\
0 \\
13\end{array}$ & $\begin{array}{l}1.2 \\
4 \\
0 \\
13\end{array}$ & $\begin{array}{c}0.5 \\
2 \\
0 \\
14\end{array}$ & $\begin{array}{l}1.0 \\
3 \\
0 \\
14\end{array}$ & $\begin{array}{c}0.6 \\
3 \\
0 \\
14\end{array}$ & $\begin{array}{l}0.2 \\
2 \\
0 \\
14\end{array}$ & $\begin{array}{l}0.0 \\
0 \\
0 \\
14\end{array}$ & 161 \\
\hline $\begin{array}{l}\text { No. Days Precipitation } \\
\text { Greater Than } \\
\text { or Equal to } 1.0 \text { in }\end{array}$ & $\begin{array}{r}\text { Avg. } \\
\text { Max. } \\
\text { Min. } \\
\text { Months of Record }\end{array}$ & $\begin{array}{l}0.1 \\
1 \\
0 \\
13\end{array}$ & $\begin{array}{l}0.0 \\
0 \\
0 \\
13\end{array}$ & $\begin{array}{l}0.1 \\
1 \\
0 \\
13\end{array}$ & $\begin{array}{l}0.2 \\
1 \\
0 \\
13\end{array}$ & $\begin{array}{l}0.5 \\
3 \\
0 \\
13\end{array}$ & $\begin{array}{l}0.7 \\
2 \\
0 \\
13\end{array}$ & $\begin{array}{l}0.5 \\
2 \\
0 \\
13\end{array}$ & $\begin{array}{l}\dot{0.1} \\
1 \\
0 \\
14\end{array}$ & $\begin{array}{l}0.2 \\
1 \\
0 \\
14\end{array}$ & $\begin{array}{l}0.0 \\
0 \\
0 \\
14\end{array}$ & $\begin{array}{l}0.1 \\
1 \\
0 \\
14\end{array}$ & $\begin{array}{l}0.0 \\
0 \\
0 \\
14\end{array}$ & $\begin{array}{l}2.4 \\
- \\
161\end{array}$ \\
\hline No. Days with Hail & $\begin{array}{r}\text { Avg. } \\
\text { Max. } \\
\text { Min. } \\
\text { Months of Record }\end{array}$ & $\begin{array}{l}0.0 \\
0 \\
0 \\
13\end{array}$ & $\begin{array}{c}0.1 \\
1 \\
0 \\
13\end{array}$ & $\begin{array}{l}0.0 \\
0 \\
0 \\
13\end{array}$ & $\begin{array}{l}0.1 \\
1 \\
0 \\
13\end{array}$ & $\begin{array}{l}0.4 \\
2 \\
0 \\
13\end{array}$ & $\begin{array}{l}0.5 \\
4 \\
0 \\
13\end{array}$ & $\begin{array}{l}0.3 \\
2 \\
0 \\
14\end{array}$ & $\begin{array}{l}0.1 \\
1 \\
0 \\
14\end{array}$ & $\begin{array}{l}0.0 \\
0 \\
0 \\
14\end{array}$ & $\begin{array}{l}0.1 \\
1 \\
0 \\
14\end{array}$ & $\begin{array}{l}0.0 \\
0 \\
0 \\
14\end{array}$ & $\begin{array}{l}0.0 \\
0 \\
0 \\
14\end{array}$ & 162 \\
\hline $\begin{array}{l}\text { No. Days with Snow on } \\
\text { Ground (Greater } \\
\text { Than or Equal to } \\
1 \text { in) }\end{array}$ & $\begin{array}{r}\text { Avg. } \\
\text { Max. } \\
\text { Min. } \\
\text { Months of Record }\end{array}$ & $\begin{array}{l}9.8 \\
23 \\
2 \\
12\end{array}$ & $\begin{array}{c}8.0 \\
19 \\
0 \\
11\end{array}$ & $\begin{array}{l}7.9 \\
15 \\
1 \\
11\end{array}$ & $\begin{array}{c}4.5 \\
13 \\
0 \\
12\end{array}$ & $\begin{array}{l}0.2 \\
2 \\
0 \\
13\end{array}$ & $\begin{array}{l}0.0 \\
0 \\
0 \\
13\end{array}$ & $\begin{array}{l}0.0 \\
0 \\
0 \\
13\end{array}$ & $\begin{array}{l}0.0 \\
0 \\
0 \\
13\end{array}$ & $\begin{array}{l}0.4 \\
4 \\
0 \\
13\end{array}$ & $\begin{array}{c}2.2 \\
18 \\
0 \\
13\end{array}$ & $\begin{array}{l}8.3 \\
25 \\
1 \\
12\end{array}$ & $\begin{array}{l}9.5 \\
25 \\
1 \\
12\end{array}$ & 50.9 \\
\hline
\end{tabular}

${ }^{a}$ Adapted by the author from data prepared by the Colorado Climatologist, Dept. of Atmospheric Science, Colorado State University, Ft. Collins, Colo. Last data Dec. 1975. 
Table 3-2. SUMMARY OF MONTHLY TEMPERATURE FOR LAKEWOOD, COLO. ${ }^{\mathrm{a}}$

\begin{tabular}{|c|c|c|c|c|c|c|c|c|c|c|c|c|c|c|}
\hline & & Jan. & Feb. & Mar. & Apr. & May & Jun. & Jul. & Aug. & Sept. & Oet. & Nov. & Dec. & Annual \\
\hline $\begin{array}{l}\text { Montrly Mean } \\
\text { Maximum Temp. } \\
(P F)\end{array}$ & $\begin{array}{r}\text { A vg. } \\
\text { Max. } \\
\text { Min. } \\
\text { Months of Record }\end{array}$ & $\begin{array}{l}44.1 \\
50.8 \\
35.5 \\
13\end{array}$ & $\begin{array}{l}46.3 \\
53.6 \\
40.7 \\
13\end{array}$ & $\begin{array}{l}59.9 \\
41.5 \\
13\end{array}$ & $\begin{array}{l}59.8 \\
65.7 \\
51.8 \\
13\end{array}$ & $\begin{array}{l}76.2 \\
64.7 \\
13\end{array}$ & $\begin{array}{l}79.0 \\
83.6 \\
71.9 \\
13\end{array}$ & $\begin{array}{l}85.4 \\
89.7 \\
81.0 \\
13\end{array}$ & $\begin{array}{l}83.6 \\
88.5 \\
80.9 \\
14\end{array}$ & $\begin{array}{l}74.7 \\
80.5 \\
66.9 \\
14\end{array}$ & $\begin{array}{l}6 E .0 \\
7 \leftarrow .3 \\
5<.1 \\
1 \leqslant\end{array}$ & $\begin{array}{l}52.6 \\
58.5 \\
42.6 \\
14\end{array}$ & $\begin{array}{l}45.4 \\
50.3 \\
39.5 \\
13\end{array}$ & 63.4 \\
\hline $\begin{array}{l}\text { Monthly Mean } \\
\text { Mininum Temp. } \\
\rho \text { F) }\end{array}$ & $\begin{array}{r}\text { Aing. } \\
\text { Max. } \\
\text { Min. } \\
\text { Months of Record }\end{array}$ & $\begin{array}{l}18.1 \\
22.9 \\
8.4 \\
13\end{array}$ & $\begin{array}{l}20.5 \\
26.2 \\
15.4 \\
13\end{array}$ & $\begin{array}{l}25.0 \\
31.4 \\
13.2 \\
13\end{array}$ & $\begin{array}{l}32.7 \\
37.5 \\
27.2 \\
13\end{array}$ & $\begin{array}{l}42.9 \\
46.9 \\
39.5 \\
13\end{array}$ & $\begin{array}{l}51.3 \\
53.5 \\
48.5 \\
13\end{array}$ & $\begin{array}{l}58.0 \\
60.8 \\
55.5 \\
13\end{array}$ & $\begin{array}{l}56.1 \\
60.5 \\
52.9 \\
14\end{array}$ & $\begin{array}{l}46.4 \\
51.4 \\
41.1 \\
14\end{array}$ & $\begin{array}{l}38.2 \\
4<.4 \\
28.9 \\
14\end{array}$ & $\begin{array}{l}26.9 \\
32.9 \\
22.0 \\
14\end{array}$ & $\begin{array}{l}19.9 \\
26.1 \\
13.7 \\
14\end{array}$ & $\begin{array}{r}36.4 \\
161\end{array}$ \\
\hline $\begin{array}{l}\text { Montbly Mean } \\
\text { Average Temp. } \\
\text { P F) }\end{array}$ & $\begin{array}{r}\text { A sg. } \\
\text { Max. } \\
\text { Min. } \\
\text { Months of Record }\end{array}$ & $\begin{array}{l}31.2 \\
36.9 \\
22.0 \\
13\end{array}$ & $\begin{array}{l}33.5 \\
39.9 \\
28.2 \\
13\end{array}$ & $\begin{array}{l}38.2 \\
45.7 \\
27.4 \\
13\end{array}$ & $\begin{array}{l}46.3 \\
51.6 \\
39.5 \\
13\end{array}$ & $\begin{array}{l}56.4 \\
61.0 \\
52.1 \\
13\end{array}$ & $\begin{array}{l}65.2 \\
68.6 \\
60.2 \\
13\end{array}$ & $\begin{array}{l}71.7 \\
74.7 \\
68.4 \\
13\end{array}$ & $\begin{array}{l}70.0 \\
73.9 \\
67.5 \\
14\end{array}$ & $\begin{array}{l}60.6 \\
65.0 \\
54.0 \\
14\end{array}$ & $\begin{array}{l}52.2 \\
58.4 \\
40.5 \\
14\end{array}$ & $\begin{array}{l}39.8 \\
45.7 \\
32.3 \\
14\end{array}$ & $\begin{array}{l}32.6 \\
37.9 \\
26.6 \\
13\end{array}$ & 49.9 \\
\hline $\begin{array}{c}\text { Degree-Deys } \\
\left.\text { (Bas: } 65^{\circ} \mathrm{F}\right)\end{array}$ & $\begin{array}{r}\text { Avg. } \\
\text { Max. } \\
\text { Min. } \\
\text { Months of Record }\end{array}$ & $\begin{array}{l}1043.7 \\
1337 . \\
862 . \\
13\end{array}$ & $\begin{array}{c}883.2 \\
1062 . \\
696 . \\
13\end{array}$ & $\begin{array}{c}822.5 \\
1157 . \\
588 . \\
13\end{array}$ & $\begin{array}{l}555.5 \\
756 . \\
394 . \\
13\end{array}$ & $\begin{array}{l}274.1 \\
407 . \\
141 . \\
13\end{array}$ & $\begin{array}{c}80.9 \\
167 . \\
8 . \\
13\end{array}$ & $\begin{array}{l}10.5 \\
59 . \\
0 . \\
13\end{array}$ & $\begin{array}{l}13.2 \\
27 . \\
0 . \\
14\end{array}$ & $\begin{array}{l}162.2 \\
330 . \\
32 . \\
14\end{array}$ & $\begin{array}{l}396.1 \\
754 . \\
227 . \\
14\end{array}$ & $\begin{array}{l}750.5 \\
972 . \\
572 . \\
14\end{array}$ & $\begin{array}{l}999.5 \\
1184 . \\
833 . \\
13\end{array}$ & $\begin{array}{c}5992.0 \\
160\end{array}$ \\
\hline $\begin{array}{l}\text { No. Lays Max. } \\
\text { Temp. Greater } \\
\text { Than or Equal } \\
\text { to } 910^{\circ} \mathrm{F}\end{array}$ & $\begin{array}{r}\text { Avg. } \\
\text { Max. } \\
\text { Min. } \\
\text { Manths of R ecord }\end{array}$ & $\begin{array}{l}0.0 \\
0 \\
0 \\
13\end{array}$ & $\begin{array}{l}0.0 \\
0 \\
0 \\
13\end{array}$ & $\begin{array}{l}0.0 \\
0 \\
0 \\
13\end{array}$ & $\begin{array}{l}0.0 \\
0 \\
0 \\
13\end{array}$ & $\begin{array}{c}0.1 \\
1 \\
0 \\
13\end{array}$ & $\begin{array}{l}3.9 \\
9 \\
0 \\
13\end{array}$ & $\begin{array}{c}8.2 \\
18 \\
2 \\
13\end{array}$ & $\begin{array}{c}4.9 \\
16 \\
0 \\
14\end{array}$ & $\begin{array}{l}0.2 \\
1 \\
0 \\
13\end{array}$ & $\begin{array}{l}6.0 \\
6 \\
61 \\
14\end{array}$ & $\begin{array}{l}0.0 \\
0 \\
0 \\
14\end{array}$ & $\begin{array}{l}0.0 \\
0 \\
0 \\
13\end{array}$ & 17.3 \\
\hline $\begin{array}{l}\text { No. Lays Max. } \\
\text { Temp. Less } \\
\text { Than or Equal } \\
\text { to } 32^{\circ} \mathrm{F}\end{array}$ & $\begin{array}{r}\text { Avg. } \\
\text { Max. } \\
\text { Min. } \\
\text { M mths of Record }\end{array}$ & $\begin{array}{c}5.5 \\
12 \\
1 \\
12\end{array}$ & $\begin{array}{l}3.2 \\
8 \\
0 \\
13\end{array}$ & $\begin{array}{l}2.4 \\
6 \\
0 \\
12\end{array}$ & $\begin{array}{l}0.4 \\
2 \\
0 \\
12\end{array}$ & $\begin{array}{c}0.0 \\
0 \\
0 \\
13\end{array}$ & $\begin{array}{l}0.0 \\
0 \\
0 \\
13\end{array}$ & $\begin{array}{c}0.0 \\
0 \\
0 \\
13\end{array}$ & $\begin{array}{l}0.0 \\
0 \\
0 \\
14\end{array}$ & $\begin{array}{l}0.0 \\
0 \\
0 \\
14\end{array}$ & $\begin{array}{c}0.3 \\
: \\
0 \\
1 \dot{z}\end{array}$ & $\begin{array}{l}1.7 \\
8 \\
0 \\
14\end{array}$ & $\begin{array}{c}4.0 \\
10 \\
0 . \\
13\end{array}$ & 17.5 \\
\hline $\begin{array}{l}\text { No. Lays Min. } \\
\text { Temp. Less } \\
\text { Than or Equal } \\
\text { to } 32^{\circ} \mathrm{F}\end{array}$ & $\begin{array}{r}\text { Avg. } \\
\text { Max. } \\
\text { Min. } \\
\text { Menth's of Record }\end{array}$ & $\begin{array}{l}27.5 \\
30 \\
24 \\
13\end{array}$ & $\begin{array}{l}25.7 \\
29 \\
23 \\
13\end{array}$ & $\begin{array}{l}24.7 \\
30 \\
18 \\
11\end{array}$ & $\begin{array}{l}14.1 \\
22 \\
6 \\
12\end{array}$ & $\begin{array}{l}2.8 \\
8 \\
0 \\
13\end{array}$ & $\begin{array}{l}0.1 \\
1 \\
0 \\
13\end{array}$ & $\begin{array}{c}0.0 \\
0 \\
0 \\
13\end{array}$ & $\begin{array}{l}0.0 \\
0 \\
0 \\
14\end{array}$ & $\begin{array}{l}1.5 \\
7 \\
0 \\
13\end{array}$ & $\begin{array}{c}7.8 \\
20 \\
\vdots \\
13\end{array}$ & $\begin{array}{l}22.1 \\
30 \\
15 \\
13\end{array}$ & $\begin{array}{l}27.4 \\
31 \\
20 \\
13\end{array}$ & 153.7 \\
\hline $\begin{array}{l}\text { No. Ilays Min. } \\
\text { Terr p. Less } \\
\text { Than or Equal } \\
\text { to } 0^{2} \mathrm{~F}\end{array}$ & $\begin{array}{r}\text { Avg. } \\
\text { Max. } \\
\text { Min. } \\
\text { Months of Record }\end{array}$ & $\begin{array}{c}4.0 \\
10 \\
0 \\
12\end{array}$ & $\begin{array}{l}0.8 \\
4 \\
0 \\
13\end{array}$ & $\begin{array}{c}0.6 \\
5 \\
0 \\
13\end{array}$ & $\begin{array}{c}0.1 \\
1 \\
0 \\
13\end{array}$ & $\begin{array}{l}0.0 \\
0 \\
0 \\
13\end{array}$ & $\begin{array}{l}0.0 \\
0 \\
0 \\
13\end{array}$ & $\begin{array}{c}0.0 \\
0 \\
0 \\
13\end{array}$ & $\begin{array}{l}0.0 \\
0 \\
0 \\
14\end{array}$ & $\begin{array}{c}0.0 \\
0 \\
0 \\
14\end{array}$ & $\begin{array}{l}0.0 \\
0 \\
0 \\
1+\end{array}$ & $\begin{array}{c}0.1 \\
1 \\
0 \\
14\end{array}$ & $\begin{array}{l}1.6 \\
7 \\
0 \\
14\end{array}$ & 7.2 \\
\hline $\begin{array}{l}\text { Highest } \\
\text { Terr.perature } \\
(\text { F) }\end{array}$ & $\begin{array}{r}\text { Tenp. } \\
\text { Y car and Day } \\
\text { Months: of Record }\end{array}$ & $\begin{array}{c}67 . \\
12\end{array}$ & $\begin{array}{c}74 . \\
197229 \\
13\end{array}$ & $\begin{array}{l}84 . \\
196328 \\
13\end{array}$ & $\begin{array}{c}86 . \\
196522 \\
10\end{array}$ & $\begin{array}{c}92 . \\
197429 \\
13\end{array}$ & $\begin{array}{c}98 . \\
196328 \\
13\end{array}$ & $\begin{array}{c}100 \\
197307+ \\
13\end{array}$ & $\begin{array}{c}98 . \\
196214 \\
13\end{array}$ & $\begin{array}{c}92 . \\
197107 \\
14\end{array}$ & $\begin{array}{c}89 . \\
196401 \\
14\end{array}$ & $\begin{array}{c}73 . \\
197313 \\
13\end{array}$ & $\begin{array}{l}70 . \\
196504+ \\
13\end{array}$ & \\
\hline $\begin{array}{l}\text { Lowest } \\
\text { Temperature } \\
\text { (F) }\end{array}$ & $\begin{array}{r}\text { Temp. } \\
\text { Year and Day } \\
\text { Month: of Record }\end{array}$ & $\begin{array}{c}-26 \\
196312 \\
13\end{array}$ & $\begin{array}{c}-6 . \\
197506 \\
13\end{array}$ & $\begin{array}{c}-11 . \\
196519 \\
13\end{array}$ & $\begin{array}{c}-1 . \\
197502 \\
13\end{array}$ & $\begin{array}{l}12 . \\
197201 \\
13\end{array}$ & $\begin{array}{c}27 . \\
197408 \\
13\end{array}$ & $\begin{array}{c}37 . \\
197217 \\
13\end{array}$ & $\begin{array}{c}41 . \\
196428 \\
14\end{array}$ & $\underset{13}{20 .}$ & $\begin{array}{c}7 . \\
13\end{array}$ & $\begin{array}{c}-2 . \\
197302 \\
14\end{array}$ & $\begin{array}{c}-17 . \\
197206 \\
13\end{array}$ & \\
\hline
\end{tabular}

${ }^{a}$ Adapted by the autior from data prepared by the Colorado Climatologist, Dept. of Atmospheric Science, Colorado State Unirersity, Ft. Collins, Colo. Last data Dec. 1975 . 
Table 3-3. SUMMARY OF MONTHLY AVERAGE RELATIVE HUMIDITY FOR ROCKY FLATS , COLO.: 1953-1976 (ELEVATION: $6000 \mathrm{ft}$ )

\begin{tabular}{|c|c|c|c|c|c|c|c|}
\hline \multirow[b]{2}{*}{ Month } & \multicolumn{3}{|c|}{$\begin{array}{c}\text { Average Relative } \\
\text { Humidity (\%) }\end{array}$} & \multirow[b]{2}{*}{ Month } & \multicolumn{3}{|c|}{$\begin{array}{c}\text { Average Relative } \\
\text { Humidity (\%) }\end{array}$} \\
\hline & $\begin{array}{l}\text { Monthly } \\
\text { Average }\end{array}$ & $\begin{array}{l}\text { Extr } \\
\text { High }\end{array}$ & $\begin{array}{l}\text { emes } \\
\text { Low }\end{array}$ & & $\begin{array}{l}\text { Monthly } \\
\text { Average }\end{array}$ & $\begin{array}{l}\text { Extre } \\
\text { High }\end{array}$ & $\begin{array}{l}\text { mes } \\
\text { Low }\end{array}$ \\
\hline Jan. & 4359 & $\begin{array}{r}26 \\
1956\end{array}$ & $\begin{array}{r}46 \\
1964\end{array}$ & July & 4550 & $\begin{array}{r}30 \\
1971\end{array}$ & $\begin{array}{r}55 \\
1962\end{array}$ \\
\hline Feb. & 4865 & $\begin{array}{r}32 \\
1959\end{array}$ & $\begin{array}{r}53 \\
1970\end{array}$ & Aug. & 4268 & $\begin{array}{r}22 \\
1955\end{array}$ & $\begin{array}{r}52 \\
1962\end{array}$ \\
\hline Mar. & 5072 & $\begin{array}{r}27 \\
1958\end{array}$ & $\begin{array}{r}57 \\
1966\end{array}$ & Sept. & 4460 & $\begin{array}{r}28 \\
1959\end{array}$ & $\begin{array}{r}53 \\
1956\end{array}$ \\
\hline Apr. & 5076 & $\begin{array}{r}34 \\
1958\end{array}$ & $\begin{array}{r}61 \\
1970\end{array}$ & Oct. & 4364 & $\begin{array}{r}24 \\
1969\end{array}$ & $\begin{array}{r}50 \\
1964\end{array}$ \\
\hline May & 5075 & $\begin{array}{r}29 \\
1957\end{array}$ & $\begin{array}{r}61 \\
1966\end{array}$ & Nov. & 4762 & $\begin{array}{r}29 \\
1972\end{array}$ & $\begin{array}{r}50 \\
1963\end{array}$ \\
\hline June & 4558 & $\begin{array}{r}34 \\
1967\end{array}$ & $\begin{array}{r}55 \\
1963\end{array}$ & $\begin{array}{l}\text { Dec. } \\
\text { Annual }\end{array}$ & $\begin{array}{l}4464 \\
4657\end{array}$ & $\begin{array}{r}26 \\
1955 \\
35 \\
1959\end{array}$ & $\begin{array}{r}48 \\
1972 \\
54 \\
1964\end{array}$ \\
\hline
\end{tabular}

a Taken from Rocky Flats monthly and annual weather summaries. 
Table 3-4. SUNSHINE AND CLOUDINESS AT DENVER, COLO. ${ }^{\mathrm{a}}$

\begin{tabular}{|c|c|c|c|c|c|c|}
\hline \multirow[b]{2}{*}{ Month } & \multicolumn{2}{|c|}{$\begin{array}{l}\text { Avg. Number } \\
\text { of Hours and } \\
\% \text { of Possiple } \\
\text { Sunshine }\end{array}$} & \multirow{2}{*}{$\begin{array}{l}\text { Avg. } \\
\text { Cloudiness } \\
\text { (0-10 tenths) }\end{array}$} & \multicolumn{3}{|c|}{$\begin{array}{l}\text { Avg. Number of Clear, } \\
\text { Partly Cloudy, } \\
\text { and Cloudy Days }\end{array}$} \\
\hline & Hours & $\%$ & & Clear & $\begin{array}{l}\text { Partly } \\
\text { Cloudy }\end{array}$ & Cloudy \\
\hline $\begin{array}{c}\text { Jan. } \\
\text { Feb. } \\
\text { Mar. } \\
\text { Apr. } \\
\text { May } \\
\text { June } \\
\text { July } \\
\text { Aug. } \\
\text { Sept. } \\
\text { Oct. } \\
\text { Nov. } \\
\text { Dec. } \\
\text { Annual }\end{array}$ & $\begin{array}{r}205 \\
201 \\
238 \\
250 \\
272 \\
310 \\
311 \\
286 \\
265 \\
242 \\
201 \\
190 \\
2971\end{array}$ & $\begin{array}{l}68 \\
66 \\
64 \\
63 \\
61 \\
69 \\
68 \\
67 \\
70 \\
70 \\
67 \\
65 \\
67\end{array}$ & & $\begin{array}{l}4.3 \\
4.6 \\
5.2 \\
5.5 \\
5.5 \\
4.6 \\
4.5 \\
4.6 \\
4.0 \\
4.0 \\
4.1 \\
4.2 \\
4.6\end{array}$ & $\begin{array}{r}14 \\
11 \\
10 \\
9 \\
8 \\
12 \\
11 \\
11 \\
15 \\
16 \\
15 \\
14 \\
146\end{array}$ & $\begin{array}{c}125 \\
116 \\
138 \\
138 \\
149 \\
144 \\
164 \\
164 \\
114 \\
105 \\
105 \\
125 \\
15267\end{array}$ \\
\hline \multicolumn{7}{|c|}{$\begin{array}{l}\text { Taken from the U.S. Department of Commerce Weather Bureau Technical } \\
\text { Paper No. 12, Sunshine and Cloudiness at Selected Stations in the United } \\
\text { States, Alaska, Hawaï, and Puerto Rico. }\end{array}$} \\
\hline \multicolumn{7}{|c|}{$\mathrm{b}_{57}$ years of record through 1948} \\
\hline $\begin{array}{l}c_{52} \text { yea } \\
\mathrm{d}_{76} \text { yea } \\
\text { sunrise } \\
\text { partly }\end{array}$ & f recorc & ough & $\begin{array}{l}48 . \\
\text { 48. Note t } \\
\text { ss the day } \\
\text { er, cloudy. }\end{array}$ & 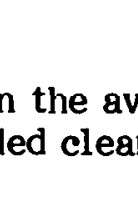 & ges & from \\
\hline
\end{tabular}

\subsection{MASTER PLAN}

A master plan has been prepared for the SERI Field Test Site to accommodate the requirements of the eighteen experiments of which SERAPH is one. The plan also provides a comprehensive infrastructure to support these experiments, including access, utilities, security, and environmental control. The site as a whole will take advantage of appropriate energy conservation concepts, and its goal is to demonstrate the effective application of solar energy technologies. The master plan establishes guidelines for energy consumption, particularly the reclamation of rejected heat from experiments (when practical). The development of the master plan was guided by the goals identified in Table 3-5. 
Table 3-5. PIELD TEST SITE MASTER PLAN GOALS

\section{FUNCTION}

To accommodate the eighteen experiments described to date.

To provide an inf rastructure of roads, utilities, environmental controls, and security for the site.

To coordinate intraexperimental functions.

To provide visibility and accessibility to the public demonstration experiments, and buffering and controlled access to the pure research experiments.

\section{FORM}

To minimize the environmental impact on the land.

To publicly enhance SERPs visual image.

To buff er adjacent neighborhoods from environmental liabilities.

To arrange the experiment's components to be sympathetic to si te contours.

\section{ECONOMY}

To avoid duplication of experiments and their components.

To maximize infrastructure efficiency.

To maximize utility service with minimum utility runs.

TIME

To provide for flexibility to accommodate change in the nature of experiments, new experiments, and expanded experiments.

\section{ENERGY}

To set an example of the "new age" in energy conservation.

To optimize the use of natural energy sources and rejected-heat reclamation for heating and cooling.

To minimize use of nonrenewable resources.

The important facts in developing the plan were primarily related to the site's location and boundary; hydrology and drainage; soils; climate; and energy/utility requirements. The needs of the eighteen experiments were satisfied given these conditions and in accord with the goals of the master plan.

A set of concepts evolved during the master planning exercise at SERI that guided the more detailed planning. These concepts are listed in Table 3-6. The master plan that evolved includes an organizational framework that can easily accommodate changes as experiments are developed. Some of the elements of the master plan are that natural drainage channels will be preserved, and large paved areas will be eschewed due to swelling soils. Straight shaft piers are recommended for foundations. A spine of services will be located at the southern boundary of the site. Public access will be controll ed and directed through the Visitor Center at the Permanent Office and visits will be limited to 


\section{Table 3-6. MASTER PLAN CONCEPTS}

SITE

Be sensitive to and respectful of the environment.

Maintain natural drainage patterns while preventing erosion.

Buff er the adjacent residential neighborhood.

Minimize cost by building on the most accessible land.

Prevent duplication of facilities.

\section{ORGANIZATION}

Develop an organization that becomes the framework for all conditions and systems.

Develop the functional areas in modular units to promote economy, flexibility and ease of access.

Develop a plan that includes options for phased growth.

Respect interexperiment functional relationships.

Centralize shared support systems, such as data acquisition, maintenance, office space, etc.

Allow for security without intimidating visitors.

\section{ENERGY}

Use the renewable resource of the sun.

Use the energy generated by the experiments at the site.

Use passive and energy-conserving designs for all structures. 
guided, supervised tours by SERI personnel. The site is environmentally isolated by a landscaped berm from a closely adjoining residential neighborhood to the south (see Fig. 3-2).

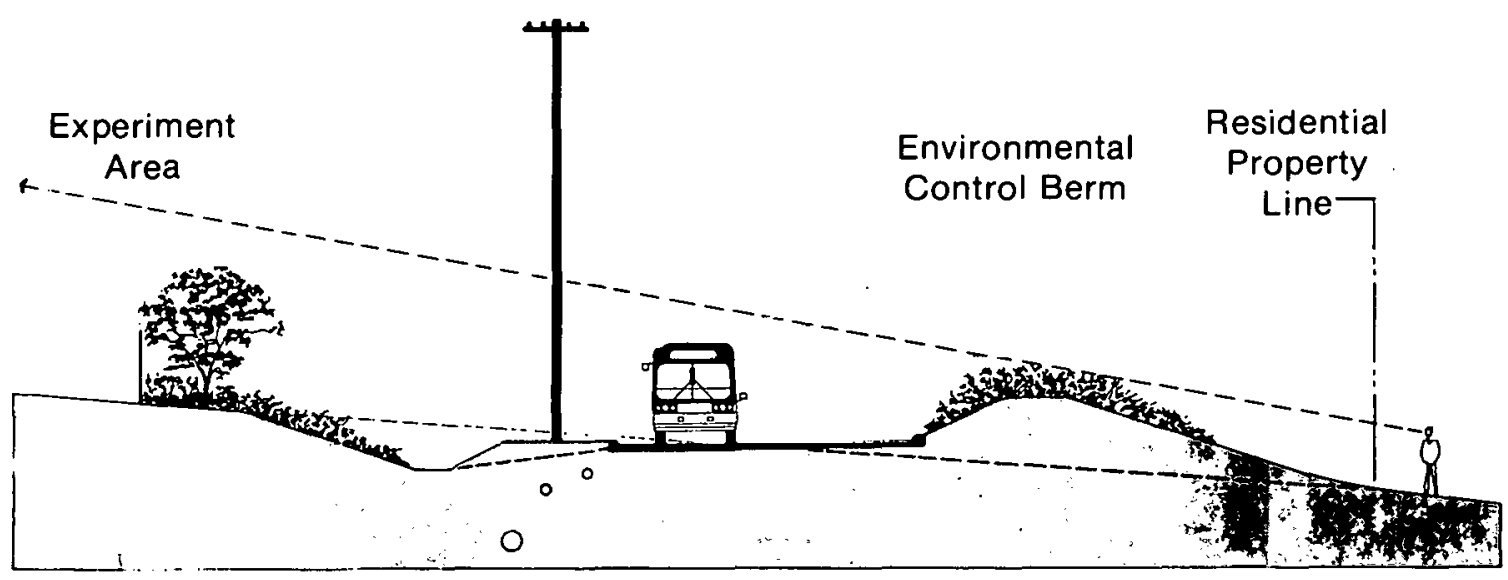

Figure 3-2. Field Test Site Buffer

The primary organizing element of the design promulgated by the master plan is the site grid. Imposed upon the site, this grid is a means of building from the utility spine a highly structured, but flexible system that organizes the access to the individual experiments, the utility service to the experiments, and the experiments themselves. By examining the size of the collector racks, structures, and the spacing of the roads and utilities, a $120^{\prime} \times 30^{\prime}(37 \times 9 \mathrm{~m})$ operational module was developed with a $15^{\prime}(4.5-\mathrm{m})$ utility and access grid connecting these modules, as shown in Fig. 3-3. Along the utility spine, utility tie-ins are located at $135^{\prime}(41-\mathrm{m})$ intervals coinciding with the $15^{\prime}(4.5-\mathrm{m})$ utility and access grids. The plan described here is essentially the development of the Field Test Site as a series of lab benches, where the buildings that house the experiments and the experiments themselves plug into a prestructured organizational system.

\subsection{SERAPH STPE}

The foregoing section describes the master planning elements that guide the implementation of SERAPH as one among the eighteen site experiments. The layout of the initial SERAPH facilities shown in Fig. 3-4 is consistent with the master plan organizational grid. The Equipment Building is positioned at the center of one of the $30^{\prime} \times 120^{\prime}$ $(9 \times 37 \mathrm{~m})$ functional areas within the grid. The Control House is adjacent to a $15^{\prime}(4.6-\mathrm{m})$ utility corridor running north-south across the site to facilitate direct access to primary electrical power, water, and sewer. The slope of South Table Mountain is such that an $8^{\prime}(2.4-\mathrm{m})$ high retaining wall is necessary behind the Equipment Building. The Equipment Building includes passive features for both heating and cooling purposes. Direct gain glazing is used on the south wall and on the skylight. Thermal storage takes place in the floor slab and in the heavy retaining wall that forms a portion of the north wall.

The areas not occupied by the structure and roadways are available for erection of collector, storage, or thermal transport hardware. These field devices are serviced from 


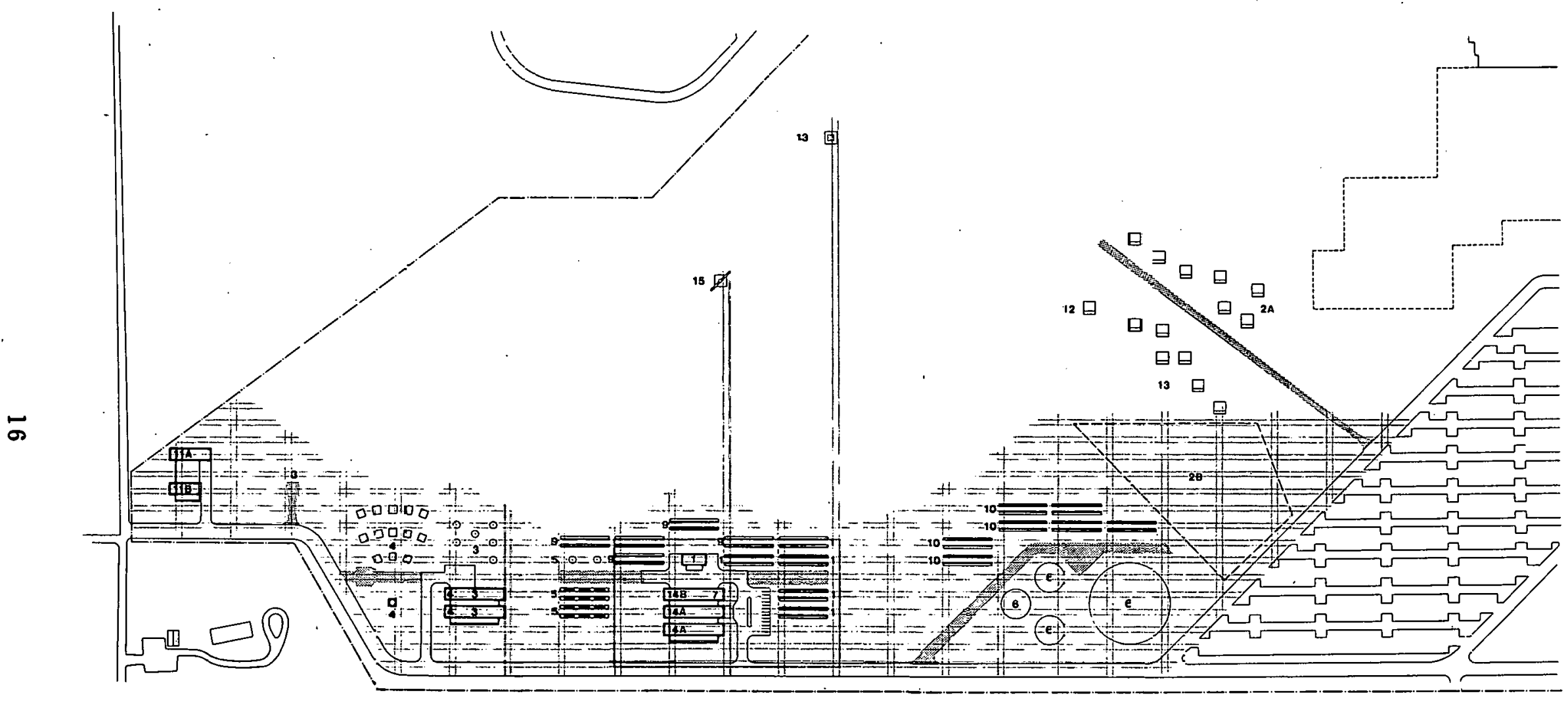

Figure 3-3. The Organizational Grid

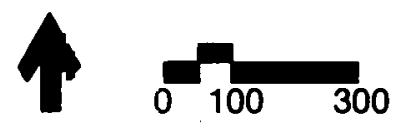




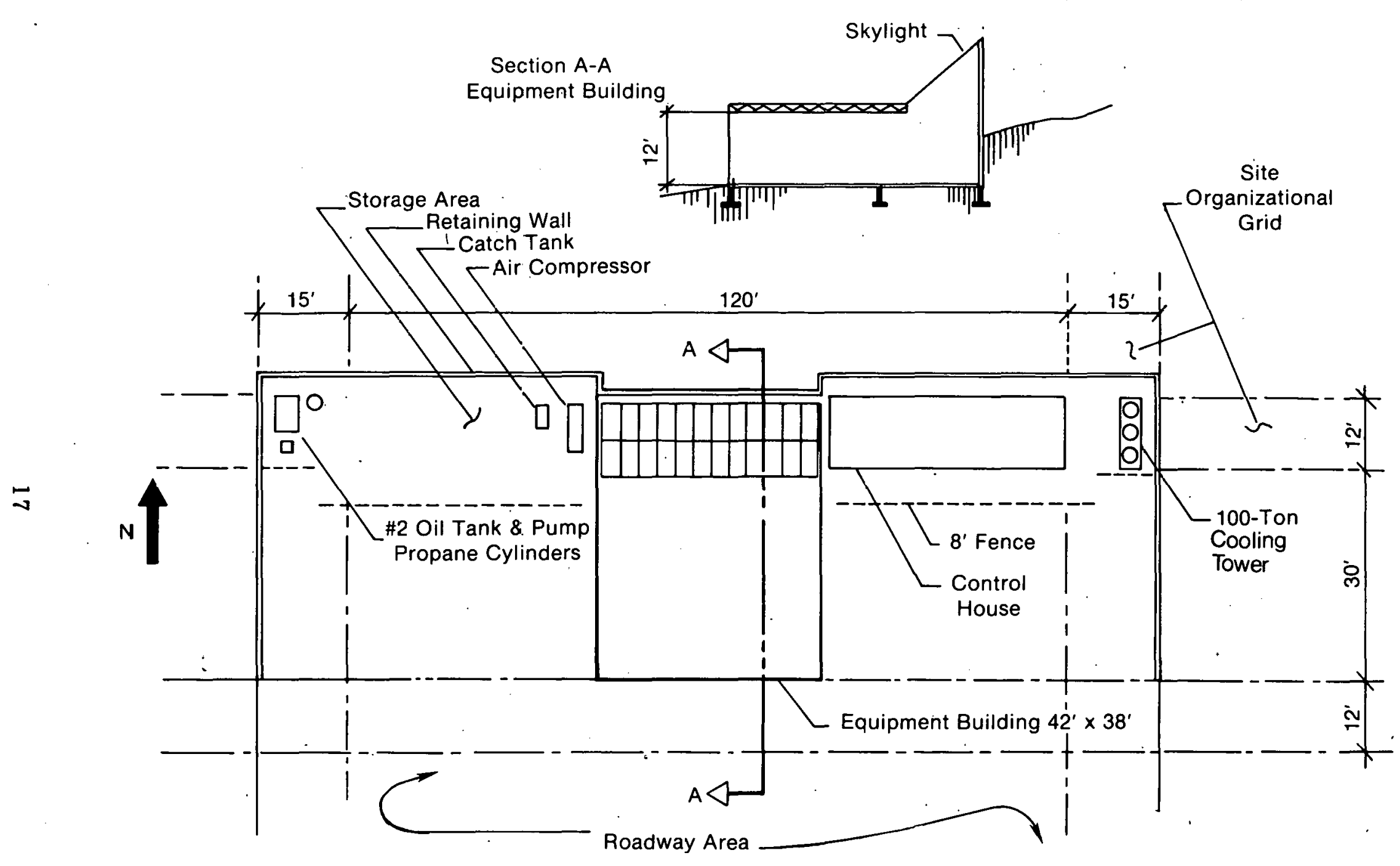

Figure 3-4. SERAPH Facility Support Elements - Plan View 
the Equipment Building via piping corridors that carry fluid lines and instrument/powercontrol signals (separate conduits) above ground. Facility control functions and data acquisition occur within the Control House. The Equipment Building houses the relatively fixed mechanical equipment needed for the facility and also provides indoor test and - work space. Sections 6.0 describes the Control House in detail.

Utilities available at the SERAPH site are identified in Table 3-7. The Control House contains a 10-kVA uninterruptible power supply (UPS) that can be used to maintain critical electrical functions for a short period following a power outage. Utilities will be buried and will not interfere with facility growth.

Table 3-7. UTHITIES AVAII ABLE AT SERAPH SITE

\begin{tabular}{|c|c|}
\hline Water & $5 \mathrm{gpm}(0.3 \ell / \mathrm{s})$ \\
\hline Electricity & $\begin{array}{r}480 \mathrm{Vac} / 3 \phi \\
100 \mathrm{~kW}\end{array}$ \\
\hline Sewer & $5 \mathrm{gpm}(0.3 \mathrm{l} / \mathrm{s})$ \\
\hline Telephone & $\begin{array}{r}1 \text { Voice } \\
\text {.. } 1 \text { Data }\end{array}$ \\
\hline $\begin{array}{l}\text { Natural Gas } \\
\text { \# } 2 \text { Fuel Oil }\end{array}$ & $\begin{array}{r}. .1500 \text { SCFM } \\
500 \text { gal }(2000 \ell)\end{array}$ \\
\hline
\end{tabular}

Distributed, linear-focus collector fields can be arranged near the Equipment Building in east-west or north-south rows with lengths acceptable up to $100-150 \mathrm{ft}(30-46 \mathrm{~m})$. The site topography requires that north-south rows be tilted toward the south by $5^{\circ}-15^{\circ}$.

Section 8.0 illustrates the installation of the first parabolic trough collector field. Vehicular access to the collector field is via east-west lateral roads on a gravel base. Water flow across the site is prevented by an interceptor flume traversing east-west on the higher, north side of the site.

The site subsurface conditions generally consist of 5 to 12 feet (1-1/2 to $4 \mathrm{~m})$ of very stiff sandy clay, with some medium-density silty sand lenses and a weathered claystonesandstone overlying a firm, to very firm, claystone or claystone-sandstone bedrock. The recommended foundation type is straight-shaft drilled piers (into the bedrock if necessary). Detailed geotechnical data for the site are available.

The presence of numerous commercial radio and television transmitting antennas on nearby Lookout Mountain subjects the si te to large RF fields. Shielding of low-level signals is necessary. Detailed electromagnetic survey data at the site is available, and a common ground grid will be provided. 


\section{SECTION 4.0}

\section{ENERGY DISTRIBUTION SYSTEM}

Routine operation of most IPH solar systems necessitates the controlled exchange of energy among the collection system, thermal storage (if present), auxiliary or primary. conventional energy source, and the process load. SERAPH, includes the Energy Distribution System (EDS) to fulfill this energy transfer role. In addition, EDS is instrumented to quantify the rate of transfer. EDS consists of thermal fluid transfer equipment and will constitute the major mechanical portion of SERAPH available on a routine basis. System testing at SERAPH will advantageously employ the multimode, versatile capabilities of EDS to thermally tie together major test elements. The inclusion of an auxiliary energy source and a controllable time-dependent load will enhance the usefulness of EDS. It will be possible to use all, a portion, or none of the EDS capabilities in a test sequence, depending upon the testing objectives. A variety of thermal fluids, including pressurized water, will also be available.

The elements of EDS are shown conceptually in Fig. 4-1. These items will be housed within the Equipment Building and will be accessible from the field areas around the building via the piping/power/instrumentation corridors. A summary of the energy transfer capabilities of EDS is found in Table 4-1.

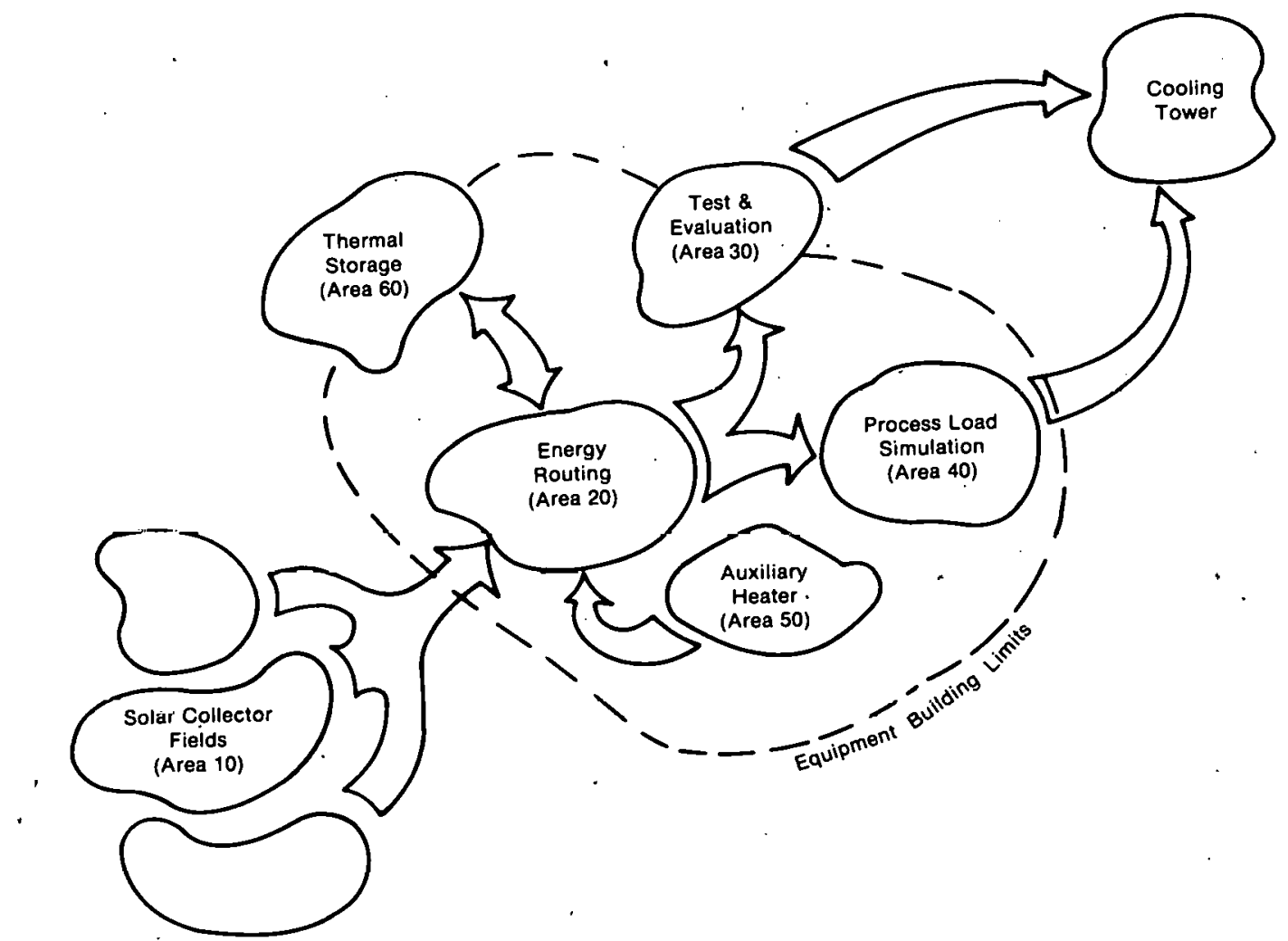

Figure 4-1. EDS Elements (Areas 20, 30, 40 \& 50) Showing Energy Flow Paths 
Table 4-1. ENERGY DISTRIBUTION SYSTEM (EDS) CAPABIITTIES

\begin{tabular}{lr}
\hline Maximum Allowable Working Pressure (MAWP) & $450 \mathrm{psig}(3100 \mathrm{kPa})$ \\
Maximum Temperature & $750^{\circ} \mathrm{F}\left(400^{\circ} \mathrm{C}\right)$ Oil \\
& $450^{\circ} \mathrm{F}\left(232^{\circ} \mathrm{C}\right)$ Water \\
Auxiliary Heat Addition & $1 \mathrm{MBtu} / \mathrm{h}(293 \mathrm{~kJ} / \mathrm{s})$ \\
Heat Rejection & $100 \mathrm{ton}(352 \mathrm{~kJ} / \mathrm{s})$ \\
Fluid Flow Rate & $100 \mathrm{gpm}(6.3 \mathrm{l} / \mathrm{s})$
\end{tabular}

The construction of EDS is modular in nature, and each of the major areas shown in Fig. 4-1 is a mechanical equipment package. Modularity serves several purposes. First, it acts to define functional difference by physical separation. Secondly, the individual packages can be shop-assembled and delivered to the site as completed units, minimizing expensive, lesser-quality field labor. American Hydrotherm Corporation was responsible for the detailed design and assembly of the three major EDS package areas. The following paragraphs describe the areas in greater detail.

Area 20 is the main pumping skid. It is responsible for primary working fluid circulation and establishing energy transf er paths. Figure 4-2 is the Piping and Instrumentation Diagram (P\&ID) for Area 20. The main circulating pump (P-200) is an Afton model ILVS 2x3-7 centrifugal pump with a 15-hp TEFC electric motor driver. Dual mechanical seals and a thermal barrier brushing within the pump minimize seal cooling, reducing parasitic losses. Nominal flow rate is $100 \mathrm{gpm}(25 \mathrm{l} / \mathrm{s})$. Flow rate control will be included in the future by throttling a fluid bypass around $\mathrm{P}-200$ or by using a variable-frequency/voltage, variable-speed driver. Thermal linking with the solar energy delivery system (Area 10) and with the storage system (Area 60) can be a direct fluid connection or an interfacial heat exchanger.

Area 50 (Heater Skid) provides a standby auxiliary energy source (see Fig. 4-3 for the P\&ID). The purpose of the $1 \mathrm{MBtu} / \mathrm{h}(250 \mathrm{~kJ} / \mathrm{s})$ oil- or natural gas-fired heater is to maintain the fluid in tank T-500 at a predetermined temperature; the fluid remains there to be available for Area 20. The Dean Brothers model-432 end-section centrifugal pump P-500 transfers heated fluid between the BS\&B Uniflux heater and tank T-500, The pressurization of T-500 establishes the pressure level throughout EDS.

Thermal energy will be transferred to the SERAPH site cooling tower via Area 40 (Support Skid). Figure 4-4 is the Area $40 \mathrm{P} \& I D$. If necessary, it will be possible to modulate the rate of energy transfer to the cooling tower by controlling the hot-side fluid flow in the shell-and-tube (Yula model HCV-6E-6DB) heat exchanger (E-400). This can be useful in reproducing specific process heat demands. Otherwise, it will be possible to transfer energy directly to the water/glycol through test hardware plugged into Area 30.

Area 30 is a piping manif old between Areas 20 and 40. Approximately $500 \mathrm{ft}^{2}\left(48 \mathrm{~m}^{2}\right)$ of space within the Equipment Building will surround the piping manifold. This area will be used for evaluating equipment designed for solar applications. (Large items will be positioned outside the Equipment Building.) Hot thermal fluid and cold water/glycol will be 


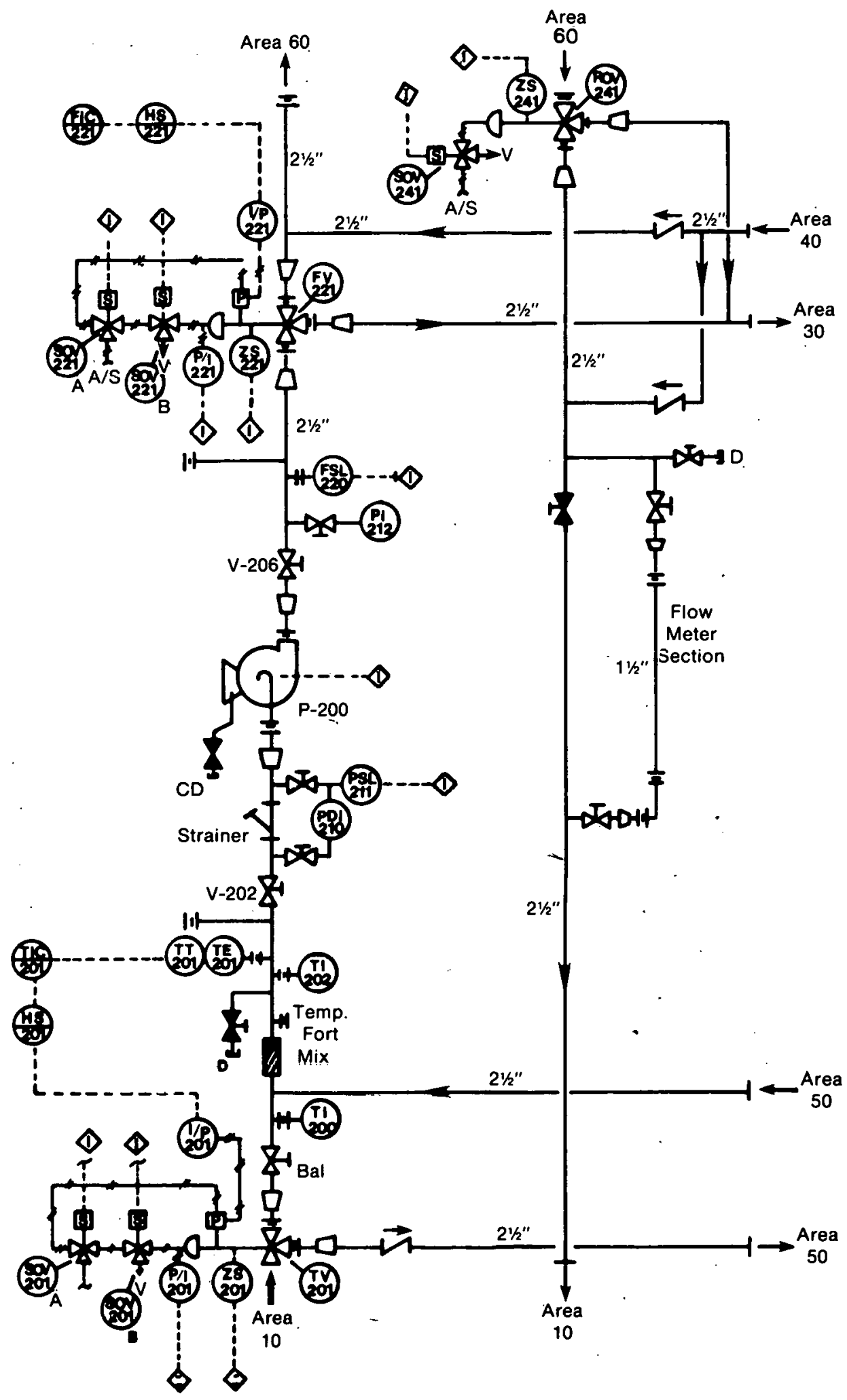

Figure 4-2. Area 20 P\&ID 


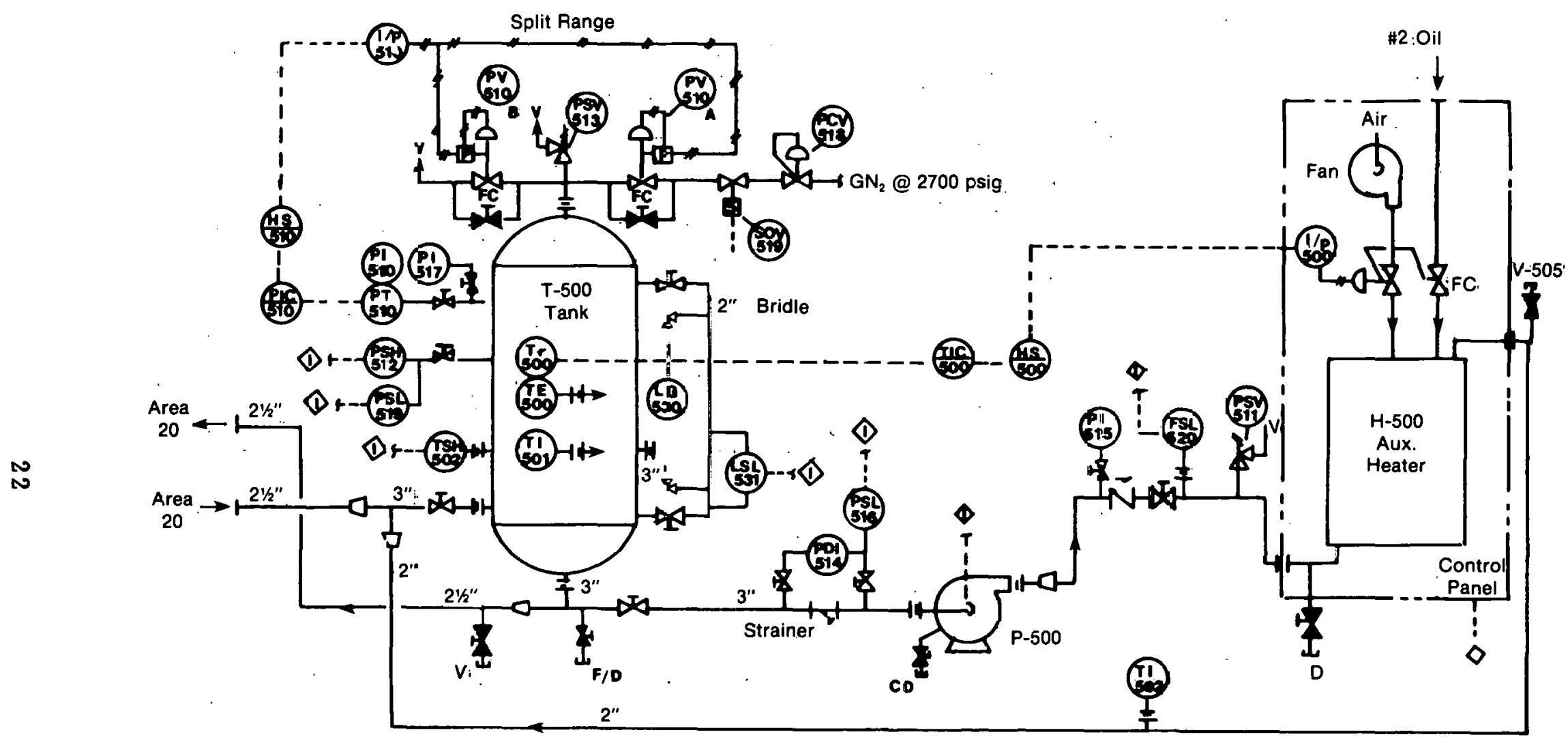

Figure 4-3. Area 50, P\&ID 


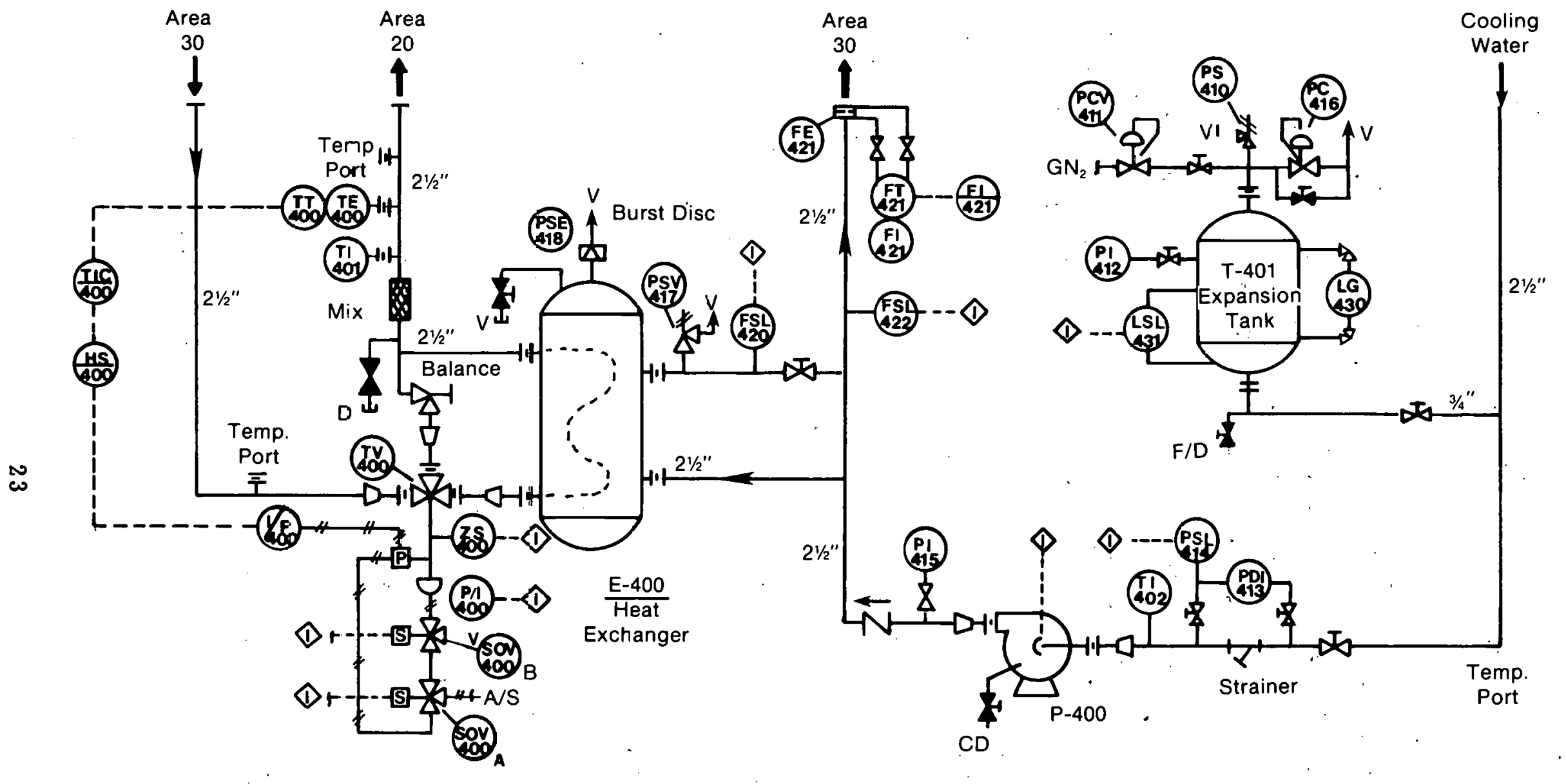

Figure 4-4. Area 40 P\&ID 
available to this test area via Area 30. In the absence of this type of test equipment, energy would flow directly from Area 20 to Area 40. Figure 4-5 shows how Areas 20, 40, and 50 (Fig. 3-4, 4-1, and 4-2) will be tied together through Area 30. The placement of the four main EDS areas within the Equipment Building is shown in Fig. 4-6. Dry compressed air at 100 psig and pump seal cooling water will be provided within the building by central distribution lines supplied by a circulating pump and air compressor package in Area 70. Tank vents, relief valves, and burst disks will be piped to an outdoor 140-gal (590- $l$ ) knockout tank via another centrally located manifold. Space will be available within the Equipment Building for items required to support collector fields or thermal storage systems such as circulating pumps and fluid expansion tanks. These same open areas are available for shop and assembly operations.

EDS is made up of welded carbon steel pipe and components. Design material selection, construction, and inspection are in conformance with Section VIII, ASME Boiler and Pressure Vessel Code, and ANSI standard B31.1 Pressure Piping. Tank hydrotesting is at 1.5-times maximum allowable working pressure and metal-jacketed calcium silicate insulation is used on hot elements. Unit heaters within the building preclude the need for freeze protection. 


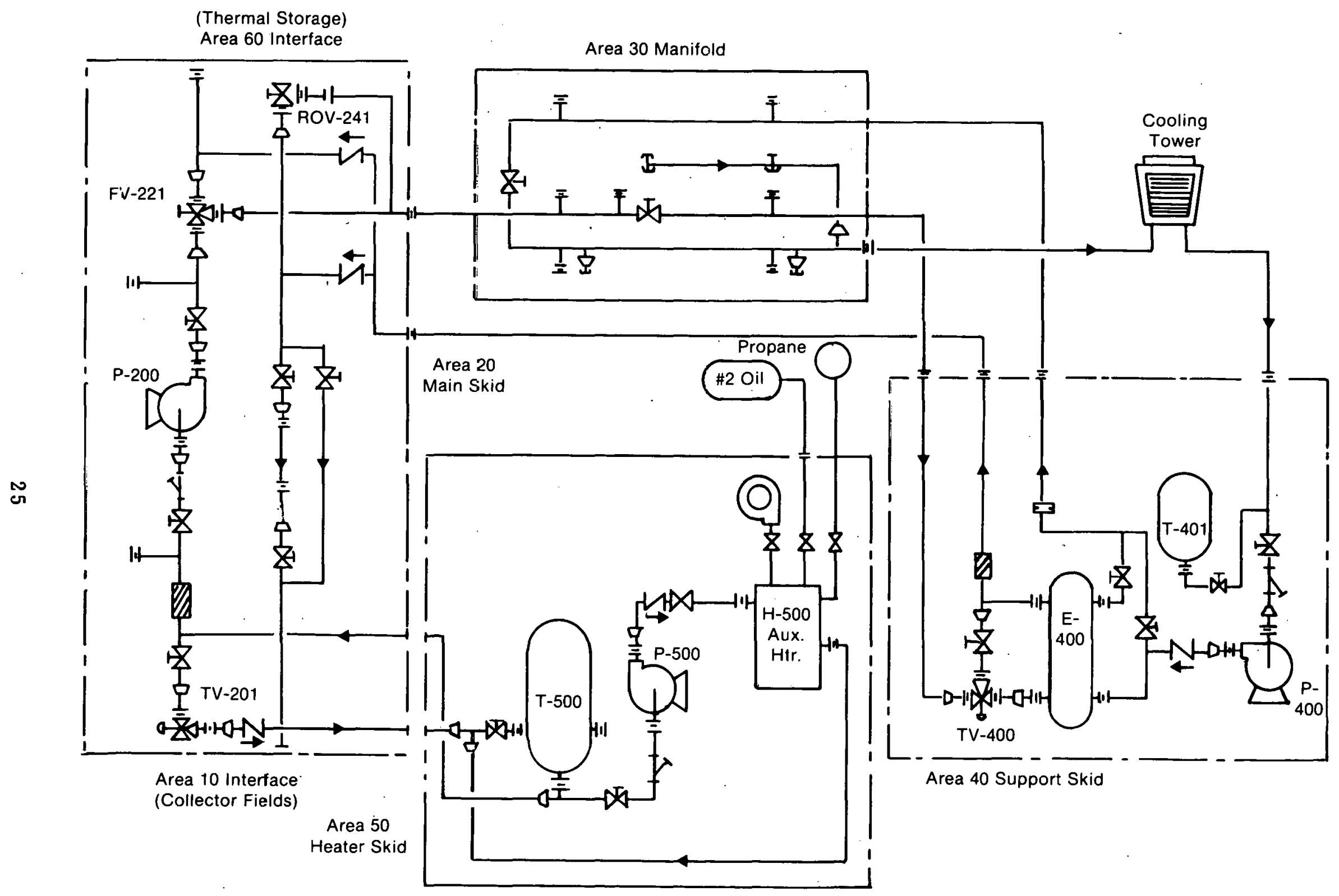

Figure 4-5. Interconnections Between Areas 20, 40, and 50 


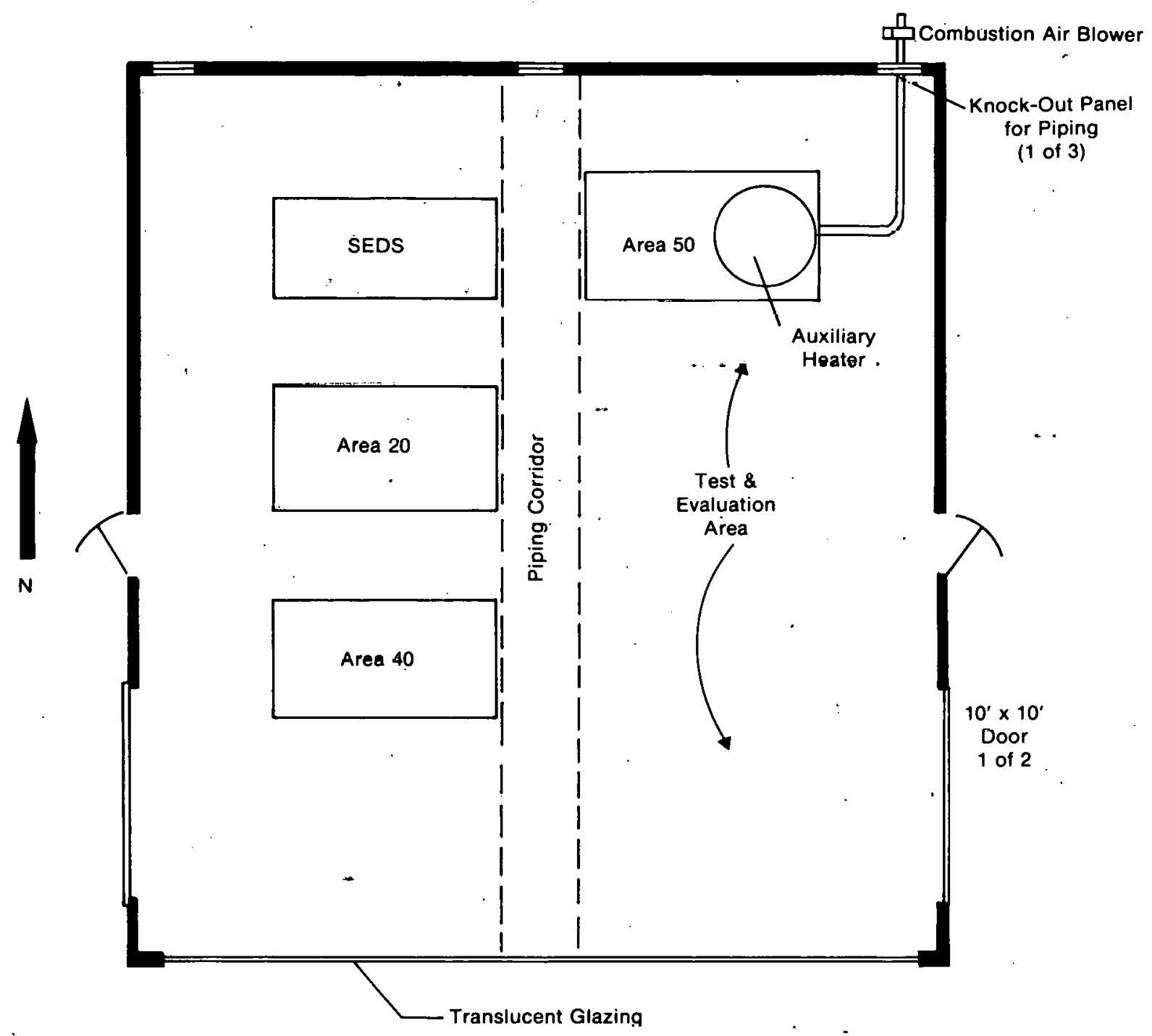

Figure 4-6. Equipment Building Layouit 


\section{SECTION 5.0}

\section{DATA ACQUISTTION}

A data acquisition system (DAS) as shown in Fig. 5-1 will be used to collect, store, and record operating data to evaluate the performance of the SERAPH equipment and developmental hardware. The DAS is a dedicated real-time digital microcomputer system consisting of the following components and peripherals:

- Digital Equipment Corp. LSI-11 central processor,

- Kennedy Model-9000 digital magnetic tape,

- Plessey Model PM-DD/11 magnetic disk storage,

- Texas Instruments Omni 810 RO printer,

- Lear Siegler Model ADM-3A CRT terminal,

- Adac 1000 Series input/output interface modules,

- IEEE-488 and RS-232 standard interface, and

- software.

The DEC LSI-11 central processor possesses a 16-bit word length and the PDP-11 series instruction set. The memory is $32 \mathrm{~K}$ words $(64 \mathrm{~K}$ bytes) semiconductor random access (RAM). The real-time clock allows the computer to be coordinated with the external world. A watchdog timer is provided to prevent a device failure from going unnoticed.

The software system, which provides an environment for program generation and execution, consists of the operating system, a FORTRAN subsystem, and a BASIC subsystem. The operating system is a single-user real-time disk operating system. The FORTRAN subsystem conforms to ANSI X3.9-1966 and includes the compiler and object time system. The BASIC subsystem is a single-user incremental version.

A total of about $22 \mathrm{~K}$ out of the $32 \mathrm{~K}$-word mem ory is available for the users' program, which permits acquisition of approximately 100 channels of data. The computer program includes conversion of raw measurements to engineering units, display of measured values to user, data recording, and limited data analysis.

The magnetic tape subsystem provides sequential access of bulk storage. The facility performance data collected by the DAS are recorded on industry-compatible magnetic tape, which in turn will be submitted to a SERI large-scale computer center for data reduction, computation, interpretation, and analysis. Local time, solar time, elapsed time, current date, and Julian date are recorded in every tape frame. The tape drive is capable of using 10-in., 2400-ft reel size and has a speed of $45 \mathrm{in} / \mathrm{s}$. The recording density is 1600 characters per in.

The DAS will be initially equipped with analog input interface modules for 64 channels of full-diff erential 4-20 mAde analog signals, and with a 64-line TTL input/output card used for shaft encoder readings (useful in monitoring angular position of tracking collectors). The $\mathrm{I} / \mathrm{O}$ interface modules can be expanded if needed in the future, provided that the DAS system capacity is not exceeded. 
$\Rightarrow 20$

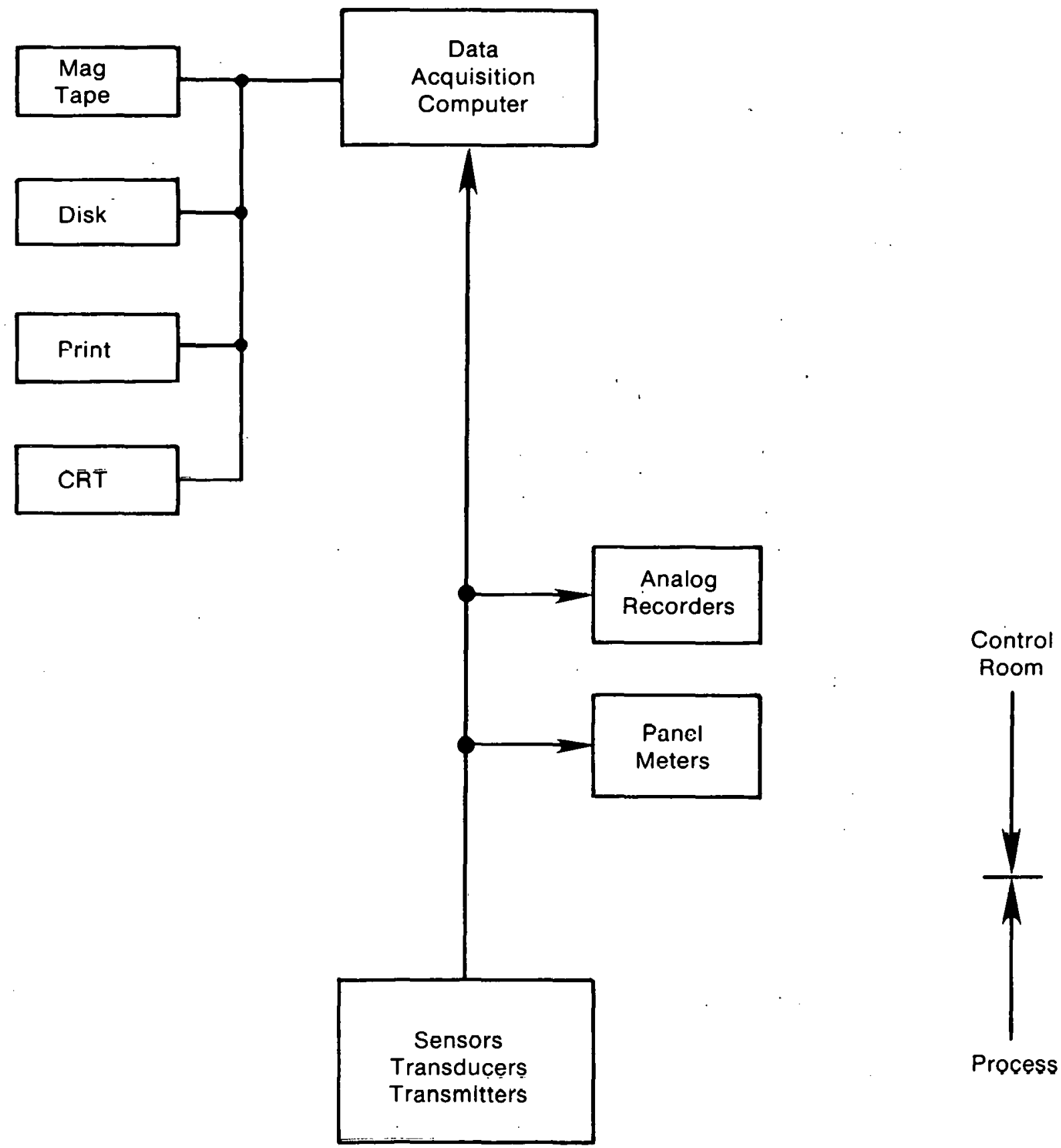

Figure 5-1. Data Acquisition System Block Diagram 
Instrument sensors, transducers, and signal transmitters for the data acquisition system are dedicated and selected so that high accuracy and reliability can be achieved. Temperatures are monitored by the resistance temperature detector (RTD) sensor and transmitter assemblies, or by the RTD sensors with the transmitters installed separately near the sensors or remotely in the control room. Pressure transmitters or transducers are used for pressure and differential pressure measurement. Liquid flow rate measurement is accomplished by turbine or orifice meters. Pulse, current, or millivolt signals are transmitted to the monitor in the Control House. To comply with industrial practice, the transmitter signals are in the form of 4-20 mAdc where applicable.

Terminal boxes, cabinets, and wiring ducts for the instrument signals are separated from those for control and power wiring to minimize problems created by electrical cross talk. A metal barrier is present within the master terminal cabinet, where the control and instrument signal wires terminate, to physically separate the 4-20 mAdc instrument signal terminal strips from the control and power terminal blocks. A completely separate, low-level signal cabinet is also provided in the Control House for RTD sensor, millivolt signal, and other low-level signal connections.

In addition to the microcomputer-based data acquisition system, analog strip chart recorders and panel meters are provided at the control panels. The analog recorders are two Texas Instruments Servo Writer III recorders, each of which has an expansion capability to six channels. The charts are 10-in. nominal and are capable of a range of speeds. The analog recorders are particularly suitable for recording transient conditions, for which continuous records are essential. Additionally, trend information can be readily observed by the operator. The panel meters provide convenient readouts of process parameters. Analog panel meters with accuracy of $1 \%$ or $2 \%$ are used. 
SERे 


\section{SECTION 6.0}

\section{- CONTROL}

The Control House, shown in Fig. 6-1, will be the centralized control center for the SERAPH facility. The SERAPH control systems will provide the operator with total manual and automatic control capabilities in the Control House for normal operations and with local manual shutdown and disabling capabilities for emergency or maintenance conditions. Except for the Auxiliary Heater (where a local control panel is present) all start-up functions will have to be initiated from the Control House, whether manually or automatically. The saf ety of SERAPH will be enhanced with this arrangem ent because a predetermined sequence and set of conditions will have to be satisfied before a start-up and during any operating period. If an emergency exists or is imminent, it will be possible to initiate shutdown without any unnecessary delay from either the Control House, Equipment Building, or the collector field area.

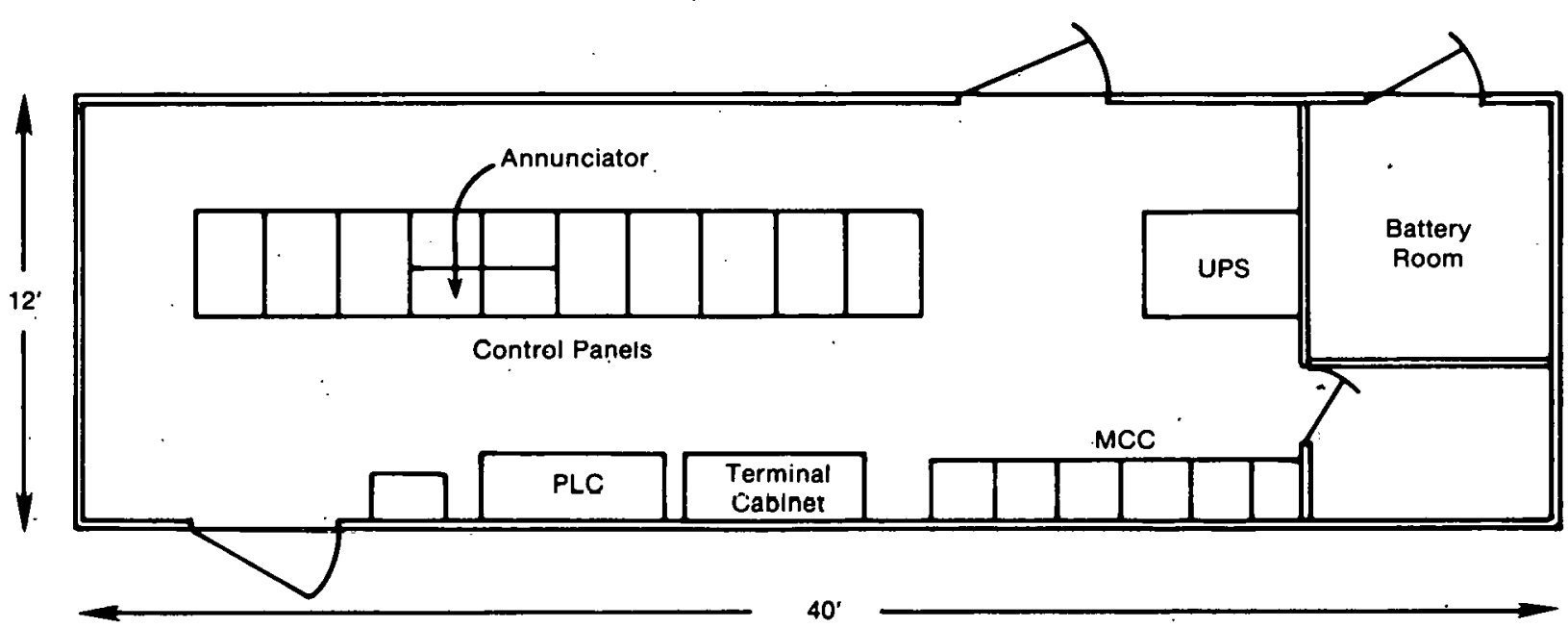

Figure 6-1. Control House Layout 
Dedicated control systems for SERAPH will be implemented in two levels (as shown in Fig. 6-2): analog controls and digital controls.

The first level of controls employs conventional analog control units and a Programmable Logic Controller (PLC) system. The control panels, consisting of electronic analog controllers, panel meters, analog recorders, indication pilot lights, graphic annunciators, pushbuttons, selector switches, etc., are the man/machine interface. When programmed, the PLC will not require the operator's attention under normal circumstances.

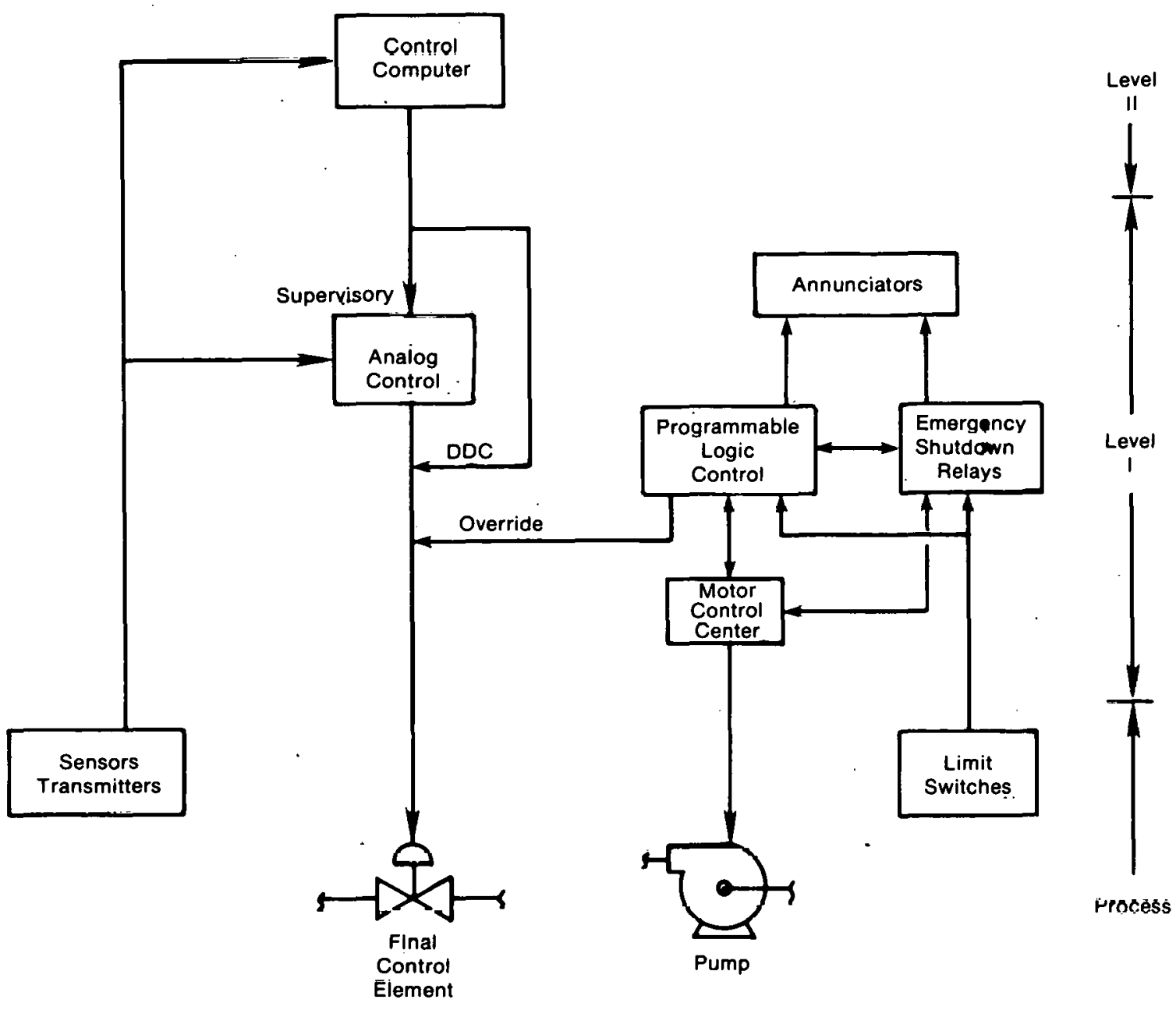

Figure 6-2. Control System Block Diagram 
The analog controllers are Moore Products Syncro 350 electronic remote set point controllers. A typical control loop using the analog controller is shown in Fig. 6-3. Each controller can operate in three modes: proportional, integral, and derivative (PID). The controller senses the deviation (error) between a process variable and its set point value, and based on the magnitude of the deviation, adjusts its output to nullify the error. The set point can be manually adjusted at the controller module, or can be automatically adjusted by a remote current source from a digital control computer. In the latter case, the controller operates in a supervisory mode. For manual controls, the controller has a manual operating mode in which an analog signal output representing the desired value is sent directly to a final control element (e.g., control valve).

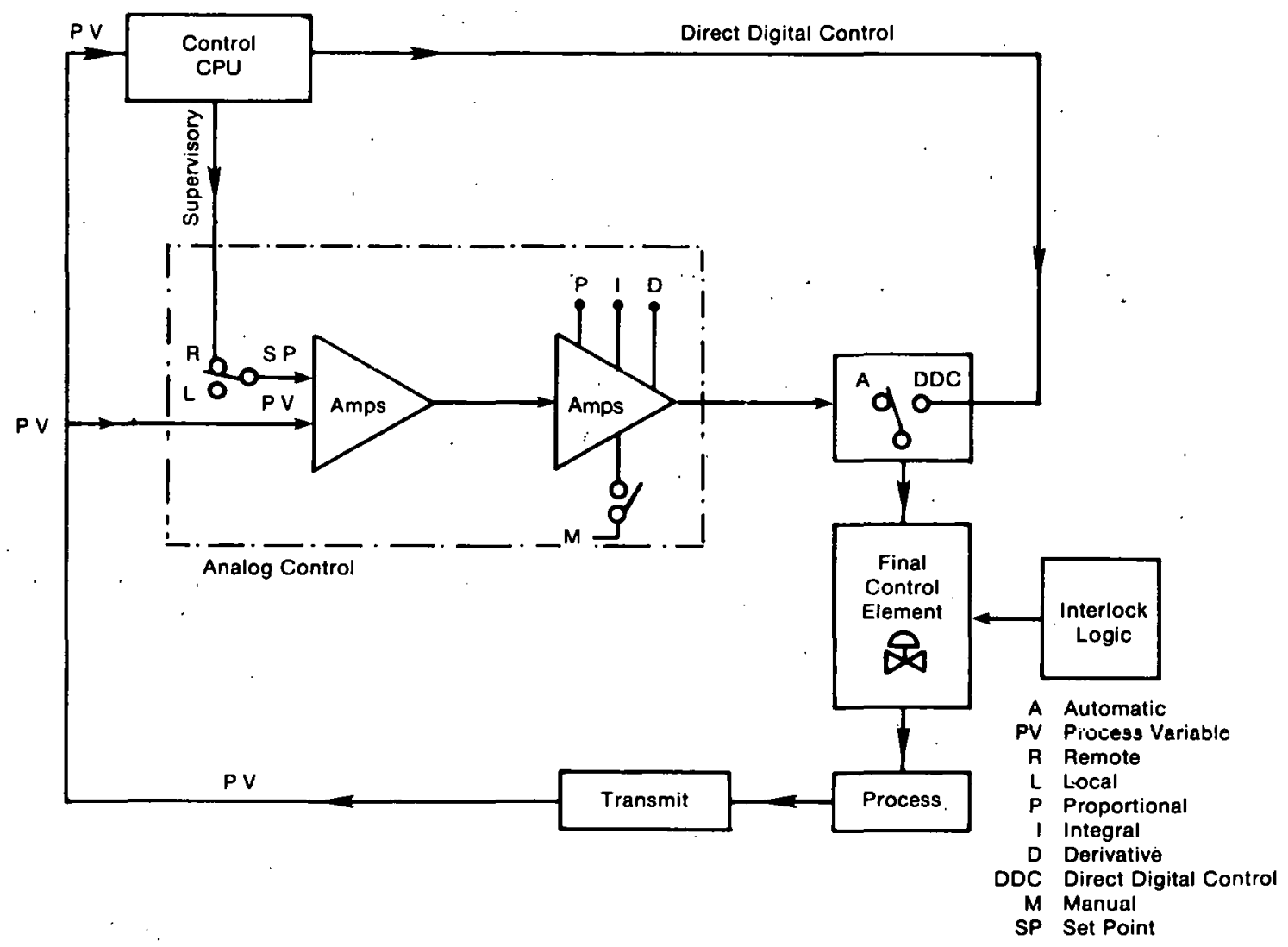

Figure 6-3. Typical Control Loop 
Using the analog controllers is considered a simple approach to gain control experience prior to implementing more sophisticated controls. Analog controls have the advantage of maintaining control loop integrity and are widely accepted by industry. The signal transmission is continuous, without the limitation imposed by the sampling periods that occur in digital control.

For logic controls such as routine start-up and shutdown, sequencing, interlock, alarming, etc., a Programmable Logic Controller system is employed. The PL.C is an off-the-shelf microprocessor-based unit whose software and input/outputs are tailored to event sequencing. The combination of a unique keyboard and sof tware enable the user to quickly establish or modify the logic required for a specific facility configuration. The PLC for SERAPH is an Allen-Bradley Bulletin 1774 PLC unit, which has an adequate expansion capability up to 1024 input/outputs. PLC programming can be accomplished with a CRT terminal plus a functional keyboard. The content of a PLC program is similar to a relay ladder diagrain.

The PLC is a solid-state device and therefore is not fail-safe. To compensate for this deficiency, hard-wired relay logic circuits are user for critical emergency shutdown functions.

Beta Products Betagraph Model-1050GA graphic annunciators will be used for graphic presentation of the SERAPH facility status. The annunciators include status lights and first-out sequence alarm annunciations in addition to a mimic display for the SERAPH process streams. The graphic display can be modified and the lights repositioned as desired when facility uses and activities change.

The second level of controls, shown in Fig. 6-1, will use a digital control computer. Digital controls will be implemented after sufficient experience has been gained from the first-level analog controls. Computer controls include supervisory control and the direct digital control (DDC). The computer continuously adjusts the set points of the PID analog controllers in the supervisory control mode. The set point values are manipulated according to algorithms programmed in the computer. The actual control actions occur in the analog controller. The computer, however, dictates the value of the process set point based upon an overall system performance model.

A selector switch is associated with each analog controller and is used to switch to direct digital control, totally bypassing the analog controller. In the DDC mode, the control actions take place in the digital computer. The computer is programmed to perform the PID control algorithms or other more sophisticated control algorithms specifically developed for SERAPH testing programs. Unlike the PID analog controllers, where the gains are manually adjusted only, the DDC gains can be changed as dictated by the algorithms and programmed in the computer sof tware. Thus, the effectiveness of the DDC controls relies heavily on the validity of control algorithms defined for the processcs.

Special control algorithms such as those for nonlinear controls, adaptive gain controls, optimal controls, etc. (which are beyond the capability of the conventional analog controllers), require the application of a digital control computer. Ultimately, it is envisioned that the digital control computer, the programmable logic controller, and the annunciators can be combined into a "Control Box" with which the CRT and its keyboard can serve as the man/machine interface for daily operations. Of course, for the Control Box to become acceptable to potential industrial users, the computer sof tware needs to be developed based on a user-oriented language and implemented in a rugged hardware configuration. 


\section{SECTION 7.0}

\section{ELECTRICAL}

A 480-volt, three-phase, four-wire, 60-Hz power system of a 100-kW capacity will be used in the SERAPH facility. The one-line diagram for the SERAPH electrical power system is shown in Fig. 7-1. In general, ac motors of $0.5 \mathrm{hp}$ and above are rated 480 volt, three phase. Motors smaller than $0.5 \mathrm{hp}$ are 115 volt, single phase. Instrumentation and control systems will use the 120 volt, $60 \mathrm{~Hz}$, or the $24 \mathrm{Vdc}$, as required. Utility systems will use the $480 / 277,208 / 120$ Vac-whichever is applicable.

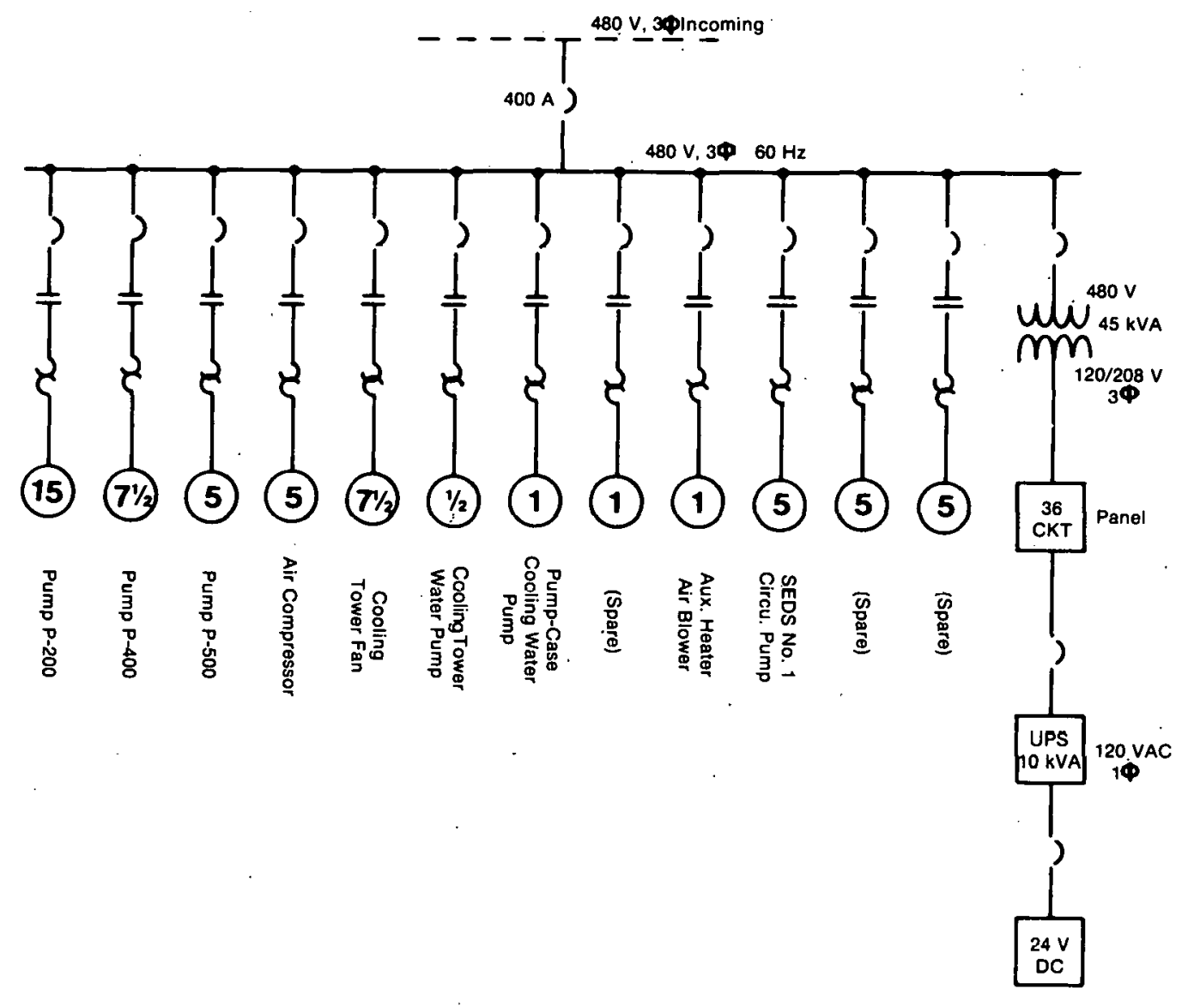

Figure 7-1. Electrical One-Line Diagram 
Most of electrical control and power distribution equipment will be in the Control House. The Control House will be a custom-engineered power control building, factorybuilt by Powell Industries. The plan layout of the Control House is shown in Fig. 5-1.

The Motor Control Center (MCC) in the Control House will be the power control and distribution center for the SERAPH facility. The main breaker frames of the MCC will include protection devices such as the ground-fault detection and tripping circuits and the ac surge arrester. Warning devices for sensing under- and over-voltage will also be included.

The MCC will be equipped with a 45-kVA stepdown power transformer to provide the 208/120-volt, three-phase/single-phase power source. Upon the occurrence of a main power failure, the control and data acquisition systems will have to be maintained for an extended period to complete safety shutdown and to protect the performance data acquired prior to interruption. For these reasone, a 10-kVA, 120-volt, 60-Hz, single-phase uninterruptible power system (UPS) of the reverse transfer type will be in the Control House. In addition to providing the uninterruptible power source, the UPS will feed the 24-Vde inverter, which in turn will provide the 24-Vde power. The UPS to be used in SERAPH is a Westinghouse Accu Pac 1 system.

Combination motor starter and control units for the motor-driven pumps will be in the MCC to enable centralized remote controls from the Control House. The initial allocation of MCC frames will provide twelve motor starter units: nine will be in use and three will be spares. Space will be available for adding two more MCC frames capable of housing 12 motor starter units of NEMA Size 1 or 2.

A variety of heat transfer fluids will be used in SERAPH. Initially, two combustible fluids from the Therminol family will be present. Therminol 66 and Therminol 60 have a flash point above $200^{\circ} \mathrm{F}\left(93.4^{\circ} \mathrm{C}\right)$ and therefore are classified as Class IIIB, per NFPA No. 321-1976, Classification of Flammable Liquids. However, as these fluids may be handled at a temperature above their flash points, the NFPA No. 30, Flammable \& Combustible Liquids Code, Chapter 5, Table 5-1, and the NFPA No. 70, National Electrical Code, Chapter 5, Articles 500 and 501 , are used as the guidelines for the electrical area classifications of the SERAPH facility. As a consequence:

- The Equipment Building is classified as Class I, Group D, Division 2.

- The Control House is classified as General Purpose, provided that the battery room in the Control House is adequately ventilated.

- The collector field is classified as General Purpose, with exceptions such as the flow meter areas, where the classification is Class I, Group D, Division 2.

- The area within 10 feet of the fuel oil storage tank is classified as a Hazardous

- Location.

Electrical practice will follow the NFPA No. 70, National Electrical Code. Where practical, the electrical wirings will be grouped (480-volt power wiring; the $120-V a c$ and 24-Vde instrumentation and control wiring; and the low-level thermocouple, RTD, and millivolt signal wiring) and physically separated.

Intrinsically safe wiring and installation will not be employed in the SERAPH facility, as the high-voltage source from the cathode ray tubes (CRT) used in the data acquisition system and the programmable controller will make it difficult to achieve a completely 
intrinsically safe practice. Instead, explosion-proof enclosures on the electrical and instrument devices will be used as required.

The Control House master terminal cabinet will terminate all control and instrument wirings from the field, Equipment Building, and within the Control House at one location. With this arrangement, a change in wiring will be easy to accomplish within the master terminal cabinet. 


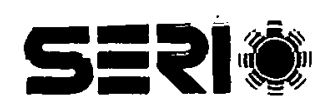




\section{SECTION 8.0}

\section{SERAPH HAZARDS/SAPETY ISSUES}

Operations at SERAPH present a range of safety issues that must be addressed in a consistent, comprehensive fashion. The responsibility for safe conditions rests with the SERI Saf ety Officer, who operates under guidelines established by DOE. The primary document that the Safety Officer relies upon in carrying out his duties is the Safe Operating Procedure (SOP) of a specific facility (e.g., SERAPH) or activity (e.g., visitor control). A SOP has been prepared for SERAPH, and it includes the following information about the basic facility:

- brief facility description;

- activities and functions taking place on a routine and nonroutine basis;

- organizations and personnel involved;

- equipment description;

- list of hazards and mitigating factors;

- precautions for operating, servicing, and repairing;

- schedule for checking saf ety equipment and saf ety drills; and

- authorized personnel.

Application of the SOP by the Safety Officer is assisted by the SERAPH Site Supervisor. The latter is intimately familiar with SERAPH equipment and operations and is therefore able to identify hazards and hazard avoidance measures more readily than the Saf ety Officer. The SOP is reviewed and updated annually or whenever significant equipment changes are made.

The usefulness of the SOP is enhanced by the inclusion of addenda with the basic document itself. The addenda may be items such as:

- equipment drawings,

- pressure saf ety analysis,

- other applicable SOPs,

- emergency procedures,

- accident analysis, and

- operational procecures.

Authorized personnel at the facility are to be familiar with the SOP and its addenda. The saf ety procedures promulgated in the SERAPH SOP conform to accepted industry norms or could be readily implemented in an industrial environment. Examples of accepted standards within industry are the National Electrical Code, National Fire Code, and the ASME Boiler and Pressure Vessel Code. Though SERAPH will be a test facility, the operations that will take place there occur (though possibly at a lesser frequency) at industrial solar installations as well. 
Separate SOPs will be prepared for major subelements of SERAPH as they become part of the facility. The basic SOP covers the EDS, its associated equipment, and the Control House and operations taking place within it. Additional SOPs will be concerned with such items as thermal storage, collector field systems, and specific test articles.

The type of hazards to personnel at SERAPH will include such items as:

- Optical: Concentrated solar radiation up to an intensity of 100 suns near focal zones of line concentrators.

- Fire: Common heat transfer oils, fuel oil, and propane (used for auxiliary heater pilot) are flammable. The presence of propane and heat transfer oils at temperatures above their flash point causes the Equipment Building and some nearby areas to recelve a Class I, Division 2, electrical classification. As a consequence, certain equipment (transmitters, switches, I/P converters, solenoids, etc.) will be placed in explosion-proof enclosures.

- Pressure: Piping, valves, pumps, and tankage may be subjected to pressures as high as 500 psig $(3450 \mathrm{kPa})$.

- Electrical: Power distribution will take place at 480 Vac within the facility (see Section 7.0).

- Windblown Debris: The SERAPH site is subject to wind gusts exceeding $100 \mathrm{mph}$ $(45 \mathrm{~m} / \mathrm{s})$.

- High Temperature: Hot circulating fluids may cause equipment (such as valve handles) to reach a temperature of $650^{\circ} \mathrm{F}\left(345^{\circ} \mathrm{C}\right)$.

- Others: Shop operations, moving colleotors, Eimultaneous operations, lightning, hail, etc.

The SOP identifies the precautions taken during design and fabrication to alleviate these hazards and the operational precautions necessary to reduce risk. Procedures and practices will be updated through the SOP review process as operational experience at the facility is aoquirod. 


\section{SECTION 9.0}

\section{FACIIITY USES}

The previous sections demonstrate that SERAPH has been configured so that it may readily adapt to a variety of uses pertinent to the IPH Program at SERI. Adaptation can take the form of mechanical equipment additions outside or inside the Equipment Building, modifications to instrumentation or control systems, and fluid exchange. These changes would take place as solar thermal technology matures and as the technical thrusts in the IPH Program evolve. Specific changes would be recommended by SERI staff within the program as well as by nonprogram individuals and organizations. Allowance has been made so that the structures themselves (Control House and Equipment Building) can be modified or expanded to meet these future needs. Mechanical equipment will be included in a modular arrangement so that it can be removed and inserted as a unit whenever practical.

This section discusses briefly several specific uses of SERAPH for projects of interest within the Solar Thermal Program structure. The first of these-the Solar Energy Delivery System-will be implemented concurrently with the buildup of the basic SERAPH facility. All others will follow and further expand the experience and information base to be established through day-to-day SERAPH operations.

\subsection{SOLAR ENERGY DEIUVRY SYSTEM (SEDS)}

A portion of the initial activity at SERAPH will be concerned with

- validation of solar thermal system performance prediction tools; and

- investigations into the interaction of solar system elements and control of this interaction.

To facilitate these objectives, a modular midtemperature (about $300^{\circ} \mathrm{C}$ ) solar energy delivery system (SEDS) will be installed at SERAPH. This will serve as the first incorporation of test equipment into SERAPH. SEDS will provide the transient thermal output so characteristic of solar systems.

In keeping with the IPH nature of SERAPH, which emphasizes fossil fuel displacement within industry, the procurement of SEDS was arranged so that it could serve as a model for industrial procurement. A thermal performance specification was prepared requiring a specific minimum net energy delivery at a specific temperature for each of four design days: these days were chosen as representative of the four seasons in Golden, Colo., where the equipment will be installed. Furthermore, this energy is to be delivered via an interfacial heat exchanger to SEDS so that the collection system remains maximally independent of the specific energy application. It is intended that this approach can be a model for future procurement purchase of an industrial solar system package.

The elements constituting the SEDS system are shown diagrammatically in Fig. 9-1. The collection system consists of line-focus parabolic troughs with a net aperture of $1956 \mathrm{ft}^{2}$ $\left(181 \mathrm{~m}^{2}\right)$. The collectors will be supported by a mechanical equipment package sitting inside the Equipment Building and containing the heat exchanger interface with EDS. 
The system has been sized to transf er a net $1 \times 10^{6} \mathrm{Btu}\left(1.06 \times 10^{6} \mathrm{~kJ}\right)$ to EDS at $385^{\circ} \mathrm{F}$ $\left(196^{\circ} \mathrm{C}\right)$ on each of the four design days. Net delivery is computed by subtracting electrical parasitics (pump driver, tracker motor, controls) and warm-up energy from the gross thermal output of the collector field. Multiple instrumentation provisions will be present in SEDS so that a detailed assessment can be made of its characteristics. The information generated will be used to validate and refine analytical models for collector subsystem performance. Several feedback control configurations will be possible for establishing system output temperature.

The parabolic trough collectors that are a part of SEDS will form three 80' (24-m) rows. These rows are positioned on the Field Test Site organizational grid as shown in Fig. 9-2. The cast concrete piers that will support these troughs are intended to be reusable. Future collert.nr fields having different module sizes will be suppurted on bridging beams that will tie together the original piers. Those piers betwcen rows, unnecessary for collector support, will form supports for simple walkways for personnel so that the soil conditions are not disturbed during observations or maintenance activities.

\subsection{COMMERCIAL CHIILER EVALUATION}

A potential use of the SERAPH facility is the testing of solar-powered chillers. Ranging in capacity from 3 to 75 tons, these units are being developed under DOE funding at Arkla Industries, Honeywell, and Carrier Corporation. Both absorption and Rankine cycle-powered units are under development. SERAPH's size is such that it would be readily compatible with chillers having ratings in the range of 15 to 25 tons-units intended for commercial operation.

A chiller would be incorporated into SERAPH for test as shown in Fig. 9-3. Solar-heated hot water delivered by the Energy Distribution Subsystem (EDS) would be supplied via a hookup with Area 30 to the generator of an absorption unit or to the boiler section in a Rankine-powered unit. The hot water would also be used to create a load condition through an isolating heat exchanger on the load side of the chiller. The required hot water temperature would be $150^{\circ}$ to $250^{\circ} \mathrm{F}\left(66^{\circ}\right.$ to $\left.121^{\circ} \mathrm{C}\right)$. Heat rejection would be accomplished at the cooling tower in SERAPH. Circulating cold water would be provided at the condenser section of the chiller (also via an Area 30 hookup) and would normally be capable of rejecting energy at the rate of approximately $800 \mathrm{MBtu} / \mathrm{h}(235 \mathrm{~kW})$ at $85^{\circ} \mathrm{F}\left(20^{\circ} \mathrm{C}\right)$.

Air conditioning units are generally rated by their manufacturers at a single operating point. Due to the variable nature of solar energy, a constant, prescribed generator inlet water temperature cannot be counted on. Therefore, it is important to know the operating characteristics of air conditioning units under transient conditions other than those stated by the manuf acturer.

Transient tests would consist of varying generator inlet temperature (GIT) as well as step-changes in the GIT (or condenser temperature) and monitoring the resultant cooling behavior. In either type of transient test, the time constant for the unit could be determined. Another kind of transient test has to do with on/off cycling. Such cycling is typical of systems having less than design loads. Long cycle-times lead to losses in capacity, since the unit has time to cool down when off, and in each restart the unit must be warmed to its design condition. 


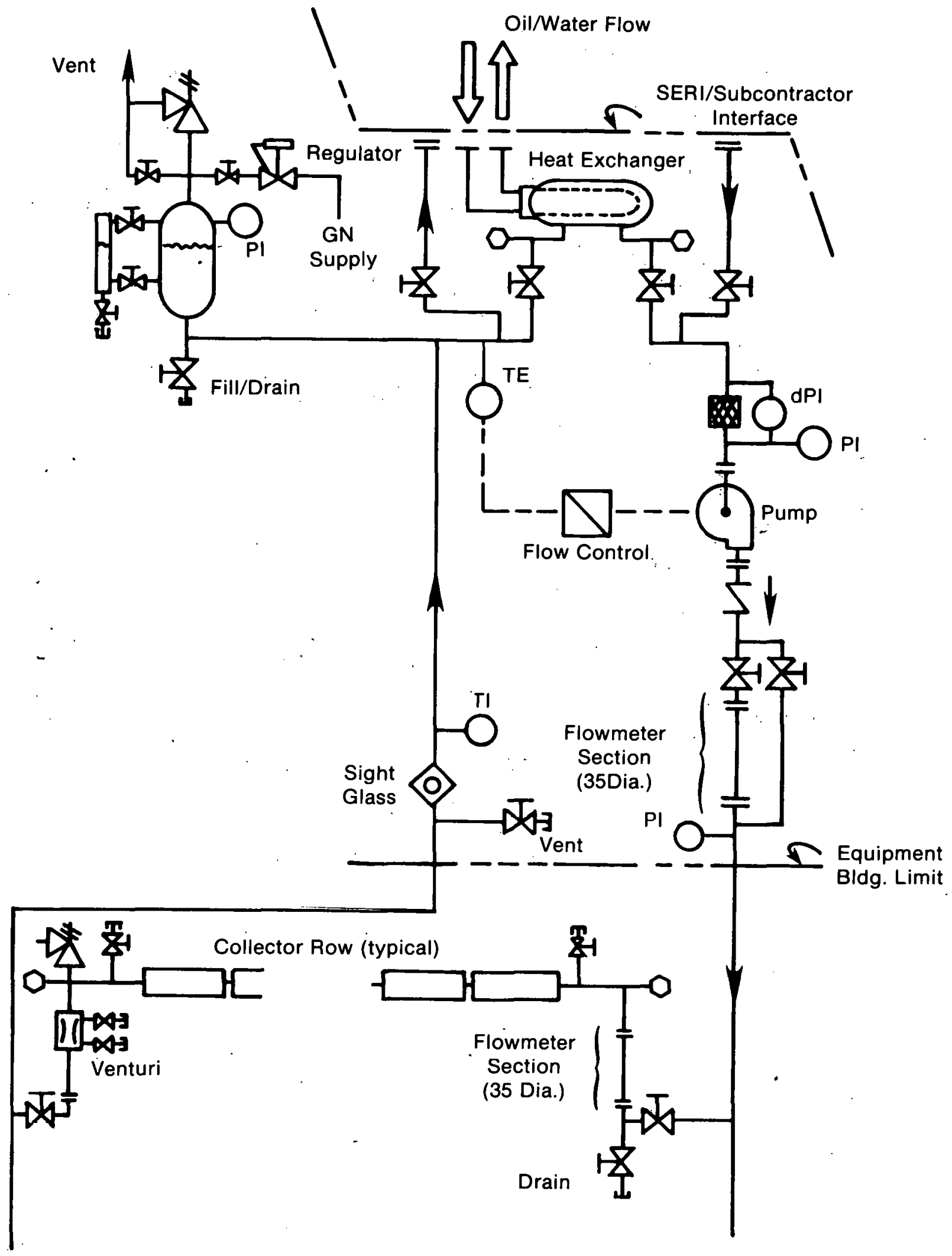

Figure 9-1. SEDS Piping and Instrumentation Diagram 


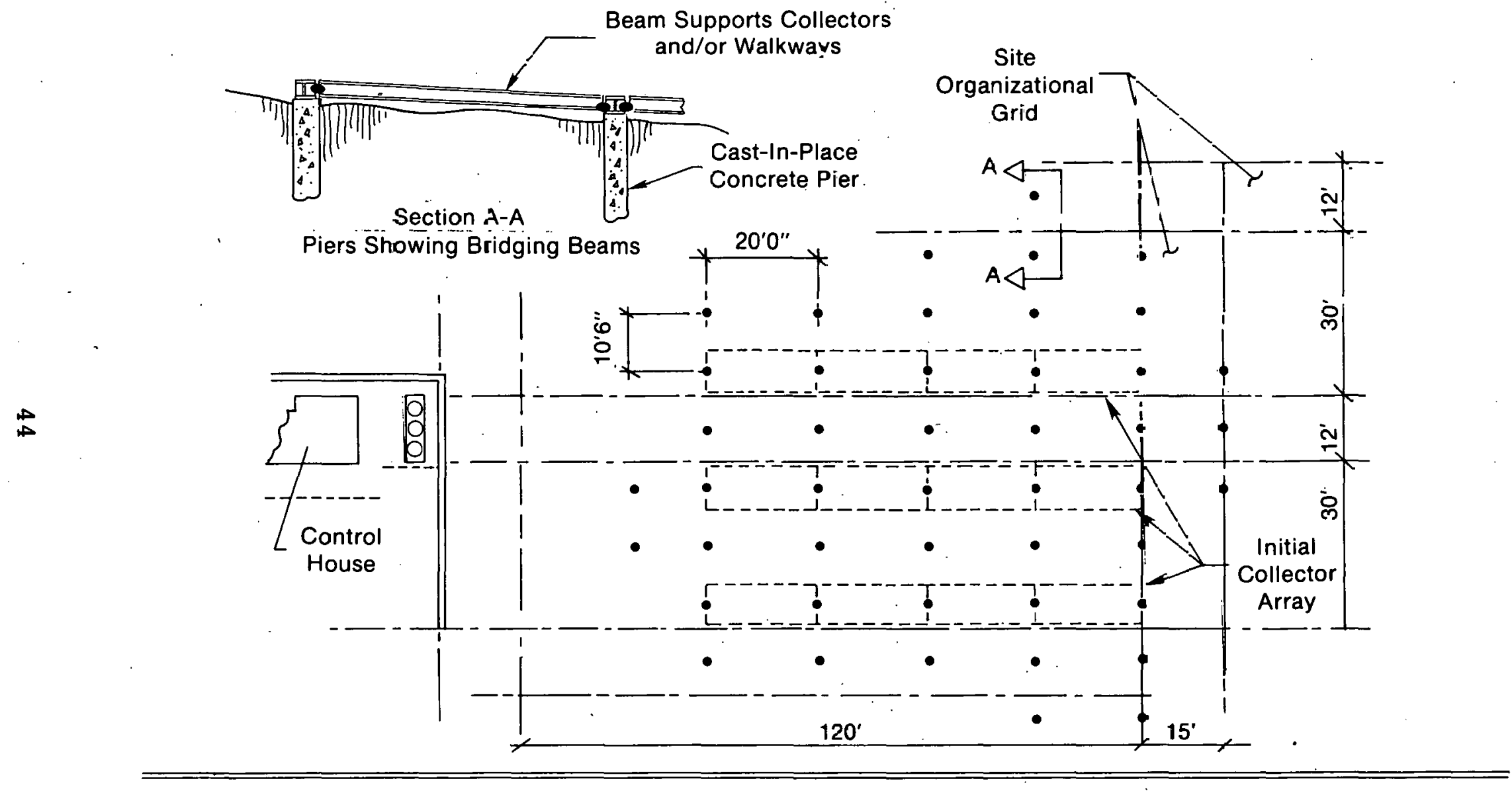

Figure 9-2. Collector Field Layout - Plan View 


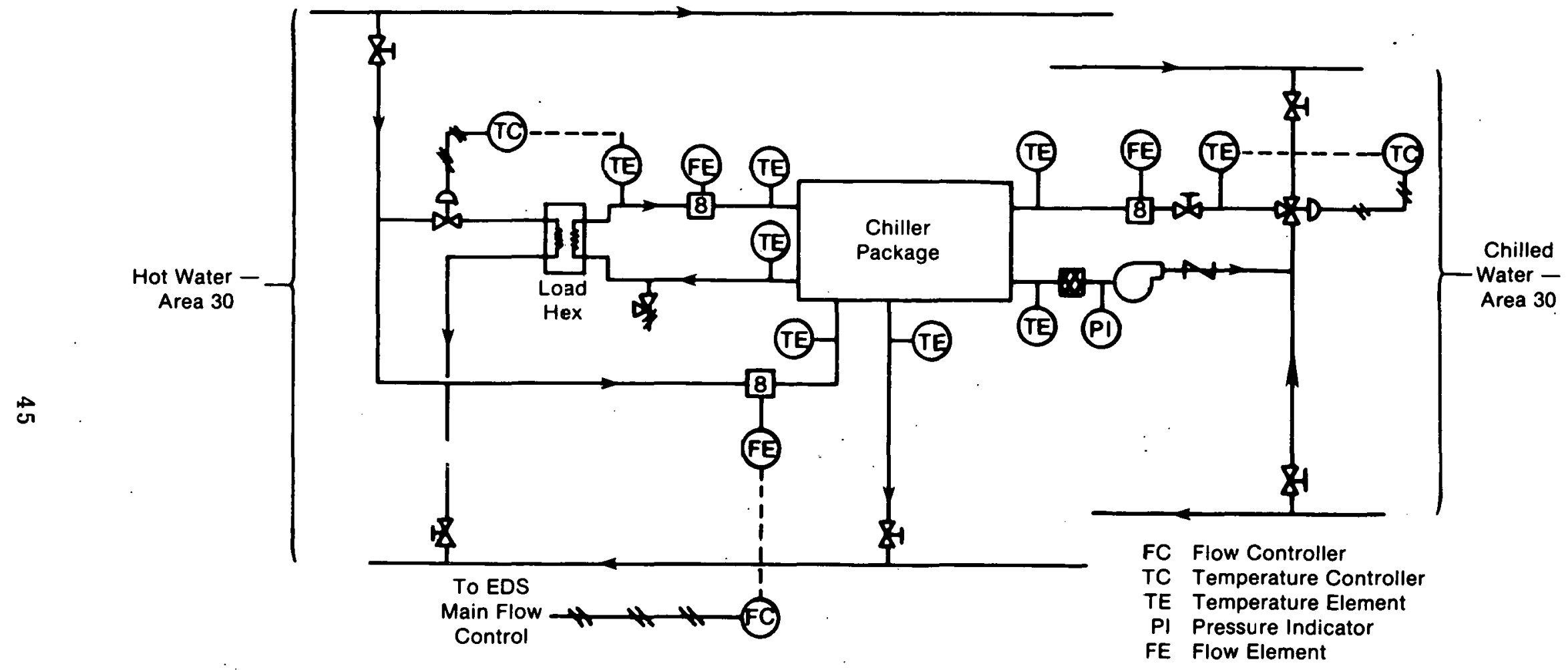

Figure 9-3. Chiller Test Configuration 
SEPI政 


\section{SECTION 10.0}

\section{CONCLUSION}

The implementation schedule for SERAPH is such that initial operations at the facility will commence in the last quarter of CY1980. Operator training, system checkout, and verification of capabilities will take place in this initial phase. Operations will follow that focus on the primary facility use objectives as enumerated in Section 2.0. Among these objectives, the first to be addressed will be thermal performance model validation and system control studies (which will also involve model validation and refinement). A detailed acceptance test procedure has been prepared that applies to the Solar Energy Delivery System (SEDS). The acceptance test is structured to reveal the net energy delivery of this solar thermal energy input package so that it can be compared readily with the suppliers' claims (when modified to account for differences between the design days and the test day).

Staffing at SERAPH will depend upon the level of test activity in progress at any given time. Normally, one or two project engineers would be involved, supported by two technicians. Technician skills will be mechanical, electrical, instrumentational, and data acquisitional in nature. The initial staff must have the capability of maintaining operations on a stand-alone basis. Support services at the Permanent Field Site will not become available until late in CY1981, when other field activities become operational. Present SERI staff will be called upon to provide design fabrication calibration and data analysis/computing assistance as required. 


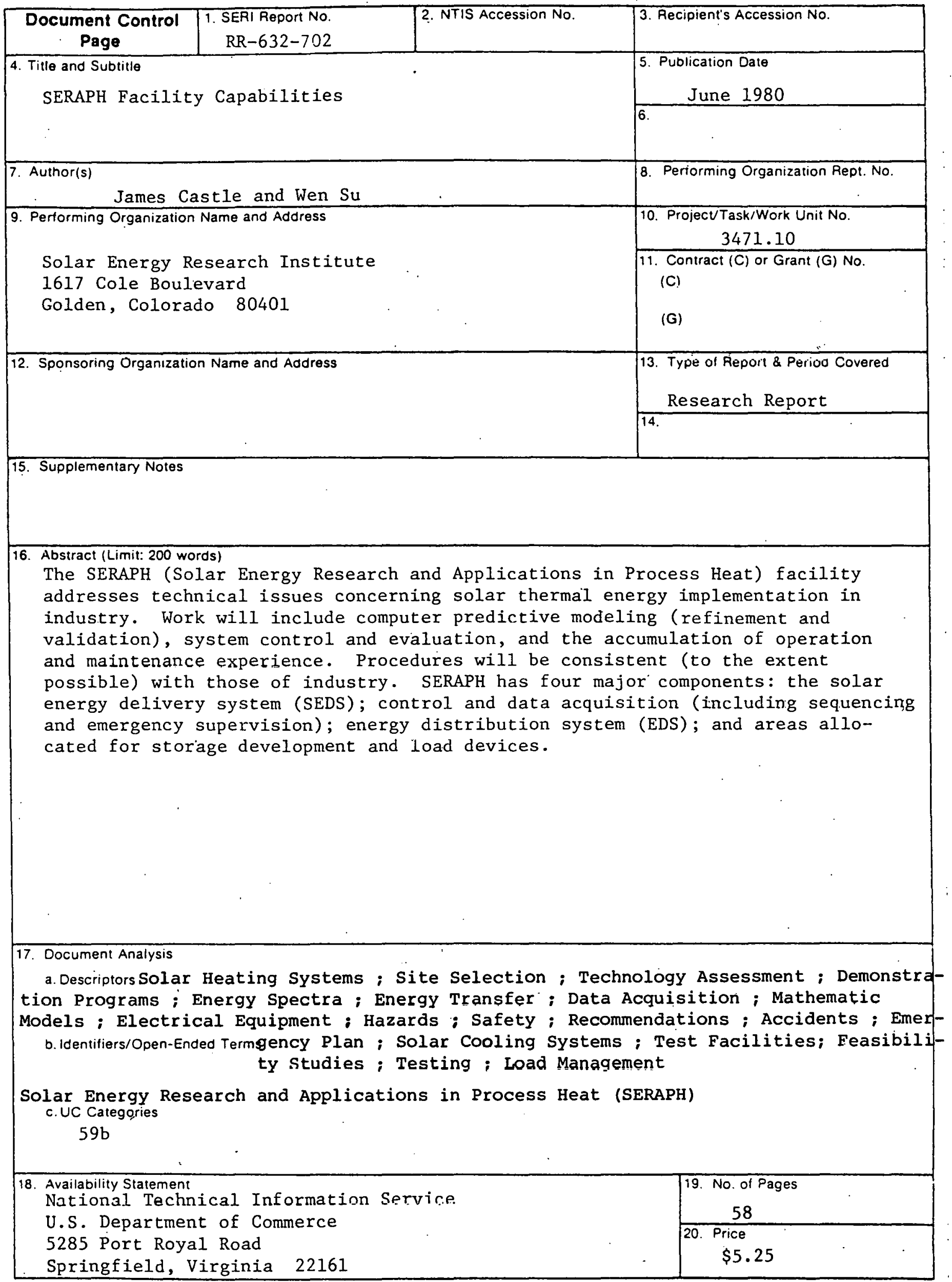

